ESAIM: PROCEEDINGS AND SURVEYS, 2018, Vol. 60, p. 1-26

Jean-François Coeurjolly \& Adeline Leclercq-Samson

\title{
MEAN FIELD GAMES: A TOY MODEL ON AN ERDÖS-RENYI GRAPH.
}

\author{
FrançOIS DELARUE ${ }^{1}$
}

\begin{abstract}
The purpose of this short article is to address a simple example of a game with a large number of players in mean field interaction when the graph connection between them is not complete but is of the Erdös-Renyi type. We study the quenched convergence of the equilibria towards the solution of a mean field game. To do so, we follow recent works on the convergence problem for mean field games and we heavily use the fact that the master equation of the asymptotic game has a strong solution.
\end{abstract}

\section{INTRODUCTION}

Mean field game theory was initiated a decade ago in independent contributions by Lasry and Lions [14-16] and by Huang, Caines and Malhamé [11,12].

The general purpose of mean field game theory is to address stochastic differential games with a large number of players interacting with one another through the empirical marginal distribution of the system, such a type of interaction being precisely called mean field. Numerous works on the theory have been dedicated to the analysis of the asymptotic formulation of the game, which is thus referred to as a mean field game. In his lectures at Collège de France, see also the lecture notes by Cardaliaguet [2], Lions [17] exposed most of the background of the analytical approach. Since then, alternative strategies, including probabilistic ones, have been suggested, see for instance [4] or the forthcoming monograph [5,6] together with the textbook [1].

One key step in the study of stochastic differential games is to prove the existence of a consensus between the players, which we also call an equilibrium. Whilst several types of equilibria are conceivable in the literature, we focus below on the notion introduced by Nash, see $[18,19]$. Basically, each player is assigned a cost - or an energy - functional depending upon a timedependent control parameter which is proper to the player (think for instance of the control as the velocity of a particle if players are particles); the cost functional is also allowed to depend on the own state of the player together with the states of the others (in agreement with the above explanation, the cost functional depends on the states of the others through the empirical distribution only when the interaction is mean field); for instance, when the player is a particle, the energy may read as the sum of the kinetic energy, which involves the own velocity of the

1 Laboratoire Dieudonné, Université Nice-Sophia Antipolis et UMR CNRS 7351, Parc Valrose, 06108 Nice Cedex 02, France.

๑) EDP Sciences, SMAI 2017 
particle, and of a potential energy, which may involve the own state of the particle together with the states of the others. We then say that a configuration (evolving with time) is a consensus between the players if there is no way for a player to decrease her/his own cost by deviating unilaterally from the consensus, namely by changing unilaterally her/his control parameter. In this framework, the rationale for regarding the mean field limit under the number of players may be explained as follows: Generally speaking, games with a large number of players are known to be of a high complexity, in the sense that solving for an equilibrium may be very costly; in case when the underlying structure is of mean field type, equilibria are expected to be of a somewhat simpler structure in the asymptotic regime. This is indeed a key feature of mean field particle systems that, asymptotically, particles are not only statistically identical but become also independent, this latter fact being usually known as propagation of chaos, see the seminal lecture notes by Sznitman [21]. Equivalently, the limiting behavior of a mean field particle system may be summed up through the dynamics of a sole representative particle interacting with its own distribution. When recast within games with a large number of mean field interacting players, propagation of chaos yields the following picture:

(1) First, an equilibrium (or a solution) of the asymptotic mean field game should consist of a flow of marginal distributions $\left(\mu_{t}\right)_{0 \leqslant t \leqslant T}$ accounting for the statistical states of the population (when in equilibrium) at any time $t \in[0, T]$, where $T$ is the time duration of the game.

(2) Second, it should suffice to focus on a typical (or representative) player (or particle) interacting with the flow $\left(\mu_{t}\right)_{0 \leqslant t \leqslant T}$ instead of regarding the whole collection of interacting players. Given $\left(\mu_{t}\right)_{0 \leqslant t \leqslant T}$, the typical player (or particle) aims at minimizing her/his own cost functional with the prescription that, therein, the empirical distribution of the system at time $t$ has been replaced by $\mu_{t}$. In words, the typical player solves a standard stochastic control problem within the environment $\left(\mu_{t}\right)_{0 \leqslant t \leqslant T}$. When the player is a particle, the point is thus to tune the velocity in order to minimize the own energy of the particle, which may be the sum of its kinetic energy and of some potential energy (which may include the interaction with the environment).

(3) Last, the Nash condition underpinning the notion of equilibrium says that the environment $\left(\mu_{t}\right)_{0 \leqslant t \leqslant T}$ forms an equilibrium if there is no incentive for the representative player to deviate -in a statistical sense- from $\left(\mu_{t}\right)_{0 \leqslant t \leqslant T}$. This holds true if and only if the best response in the optimization problem under the flow $\left(\mu_{t}\right)_{0 \leqslant t \leqslant T}$-as addressed in point (2) right above fits $\left(\mu_{t}\right)_{0 \leqslant t \leqslant T}$ exactly, namely if the collection of marginal laws of the optimal state is $\left(\mu_{t}\right)_{0 \leqslant t \leqslant T}$ itself.

In short, an equilibrium is thus a fixed point for an application mapping a flow of statistical distributions onto another flow of statistical distributions. Of course, the challenge is then to guarantee the existence of such a fixed point; this requires first a tractable characterization of it, which may be of analytical or probabilistic nature.

From the analytical point of view, fixed points may be characterized as solutions of a forwardbackward system made of two coupled evolution equations, a forward Fokker-Planck equation and a backward Hamilton-Jacobi-Bellman equation: By forward equation, we mean that the boundary datum is given at the initial time, and, by backward equation, we mean that the boundary datum is given at the terminal time; saying that the two equations are coupled is the same as saying that each of the two equations depends upon the solution of the other one. As explained below, the fact that the two coupled equations have opposite directions makes the whole rather difficult to solve. In our setting, the resulting system is usually referred to 
as the mean field game system: Therein, the backward equation accounts for the optimization part in the search for an equilibrium -see point (2) in the above description of a mean field game--, whilst the forward one describes the evolution of the equilibrium $\left(\mu_{t}\right)_{0 \leqslant t \leqslant T}$ under the Nash condition (3). The probabilistic approach obeys more or less the same principle, as it also relies on a forward-backward system, but of a somewhat different structure. Precisely, this forward-backward system reads as a forward-backward stochastic differential equation of the McKean-Vlasov type. The forward component provides the form of the optimal trajectories of the stochastic optimization problem -again, this is (2) above- whilst the McKean-Vlasov condition enforces the fixed point constraint following from the Nash condition -see (3)-. Whatever the approach, the key problem is to sort out the forward-backward structure arising in the characterization. It is indeed known that the Cauchy-Lipschitz theory for systems of coupled forward-backward differential equations only applies when the time duration $T$ over which the system is set is small enough. Rephrased in our setting, this says that, when $T$ is fixed, equilibria of mean field games cannot be systematically constructed by a contraction argument. This is in stark contrast with the theory of differential equations with either one initial or one terminal boundary datum, for which Picard's theorem applies as long as the coefficients are Lipschitz continuous. Most of the time, the construction of an equilibrium to a mean field game thus requires another method; for instance, it may be based on a fixed point theorem without uniqueness. Also, as the mapping driving the fixed point equation may not be a contraction, uniqueness for mean field games is known in very few cases; for example, it holds true when the coefficients satisfy specific monotonicity conditions, which we shall illustrate below.

Although there is no specific reason for expecting it to hold true in full generality, uniqueness is an important question. When it fails, it may be a very difficult question to select one of the equilibria. Also, uniqueness may be very useful for justifying the convergence of equilibria of games with finitely many players towards solutions of mean field games. As a matter of fact, proving the convergence of finite player games, which means proving the convergence of the equilibria as the number of players tends to infinity, is a difficult problem, which may be addressed in two different ways according to the precise notion of Nash equilibrium which is used. In the finite player game, Nash equilibria may be indeed taken over either open or closed loop controls: Roughly speaking, an equilibrium is said to be over open loop controls if the players in equilibrium are blind to the deviation chosen by any unilaterally deviating player; in contrast, an equilibrium is said to be over closed loop controls if each player computes her/his own control as a function of the states of all the players; when they do so, the players in equilibrium update their controls when one of them is deviating. Regarding the convergence problem, the picture is then as follows. When the finite player equilibria are taken over open loop controls, compactness arguments, without any need for asymptotic uniqueness, may be used, see for instance $[10,13]$; however, this strategy fails when equilibria are computed over controls in closed loop form. In the latter case, the only strategy that has been known so far for tackling the convergence problem requires uniqueness, see [3]. The idea for doing so goes back to another key object due to Lions, which is known as the master equation. Whenever uniqueness holds, the aforementioned forward backward system used to characterize the solution of the mean field game (whatever the approach is analytical or probabilistic) may be regarded as the characteristics of a nonlinear PDE set on the space of probability measures. This latter PDE is precisely the master equation. Whenever the master equation has a classical solution, say $\mathcal{U}$, see for instance $[3,8]$ for solvability results in that direction, convergence may be proved by letting $\mathcal{U}$ act onto the empirical distribution of the equilibria of the finite player game. 
The purpose of this paper is to revisit all these facts, but in a somewhat different framework from what is usually addressed in the literature. Whilst the interactions in the finite player system that have been considered so far are always defined on the complete graph, we here assume that some of the particles do not directly interact with one another and, more precisely, that the connections between all of them are given by the realization of an Erdös-Renyi graph with a non-trivial parameter. In order to simplify, we focus on a toy model only, for which the structure of the asymptotic game is not too complex. In this framework, we prove that the limiting problem is also a mean field game. Taking advantage of the simple form of the model, we manage to simplify the master equation and to show directly, with an affordable price, that it has a classical solution. Last, we address the convergence of the equilibria of the finite player system when the realization of the graph is frozen. To simplify, we assume that equilibria are taken over open loop controls, but, in order to exemplify the notion of master equation, we make use of this latter one to pass to the limit, as explained above.

We present the model in Section 2. The asymptotic game is investigated in Section 3 . The convergence problem is addressed in Section 4.

\section{A TOY MODEL}

First, we introduce the finite player game we alluded to in the introduction. For the sake of simplicity, all the particles we consider below live in $\mathbb{R}$.

\subsection{Particle system}

In this paragraph, we are given a collection of $N$ real-valued Brownian motions $\left(\left(W_{t}^{i}\right)_{0 \leqslant t \leqslant T}\right)_{i=1, \cdots, N}$, where $T$ is some finite time horizon, the value of which is fixed throughout the article, and $N$ is an integer. These processes are assumed to be constructed on a probability space $(\Omega, \mathcal{F}, \mathbb{P})$. The filtration generated by the noises and possibly augmented with an initial $\sigma$-field $\mathcal{F}_{0}$, independent of $\left(\left(W_{t}^{i}\right)_{0 \leqslant t \leqslant T}\right)_{i=1, \cdots, N}$, is denoted by $\left(\mathcal{F}_{t}\right)_{0 \leqslant t \leqslant T}$.

With each index $i \in\{1, \cdots, N\}$, we associate a particle (or a player). At any time $t$, the dynamics of player $i$ are driven by some instantaneous velocity, which we denote by $\alpha_{t}^{i}$, and are forced by the Brownian fluctuation $d W_{t}^{i}$, which is proper to player $i$. The dynamics are thus of the following linear form:

$$
d X_{t}^{i}=\alpha_{t}^{i} d t+d W_{t}^{i}, \quad t \in[0, T]
$$

We stress the fact that the term $\alpha_{t}^{i}$ is not a priori fixed. As explained below, the player may tune it in order to minimize some criterion. For that reason, the process $\left(\alpha_{t}^{i}\right)_{0 \leqslant t \leqslant T}$ is called a control. It is required to be $\left(\mathcal{F}_{t}\right)_{0 \leqslant t \leqslant T}$ progressively-measurable and to satisfy the square-integrability condition

$$
\mathbb{E} \int_{0}^{T}\left(\alpha_{t}^{i}\right)^{2} d t<\infty
$$

Last, the initial conditions $\left(X_{0}^{i}\right)_{i=1, \cdots, N}$ are assumed to be $\mathcal{F}_{0}$-measurable, square-integrable, independent and identically distributed. 


\subsection{Interactions between the particles}

With each particle, say $i \in\{1, \cdots, N\}$, we associate a cost functional $J^{i}$ depending upon all the control processes $\left(\left(\alpha_{t}^{i}\right)_{0 \leqslant t \leqslant T}\right)_{i=1, \cdots, N}$. It takes the following form:

$$
\begin{aligned}
& J^{i}\left(\left(\left(\alpha_{t}^{j}\right)_{0 \leqslant t \leqslant T}\right)_{j=1, \cdots, N}\right) \\
& =\mathbb{E}\left[\frac{1}{2}\left(c_{g} X_{T}^{i}-\int_{\mathbb{R}} g(x) d \bar{\mu}_{T}^{N, i}(x)\right)^{2}\right. \\
& \left.\quad+\int_{0}^{T}\left[\frac{1}{2}\left(c_{f} X_{t}^{i}-\int_{\mathbb{R}} f(x) d \bar{\mu}_{t}^{N, i}(x)\right)^{2}+\frac{1}{2}\left(\alpha_{t}^{i}\right)^{2}\right] d t\right],
\end{aligned}
$$

where $c_{f}$ and $c_{g}$ are two constants, $f$ and $g$ are two functions from $\mathbb{R}$ into itself and $\left(\bar{\mu}_{t}^{N, i}\right)_{0 \leqslant t \leqslant T}$ is a collection of probability measures, the definition of which is given next. Roughly speaking, each $\bar{\mu}_{t}^{N, i}$ accounts for the empirical distribution at time $t$ of the so-called particles that are connected to $i$; we make clear the definition of these connections right below. The cost functional $J^{i}$ thus involves three kinds of terms: In the integral from 0 and $T$, the last term may be understood as the kinetic energy of particle $i$ if we regard the control $\alpha_{t}^{i}$ at time $t$ as an instantaneous velocity; the term on the second line and the first term in the integral from 0 and $T$ describe some cost related to the interactions between the particle $i$ and the particles that are connected to it; according to the preliminary discussion in introduction, we may call this interaction cost a potential energy. In this potential energy, the term $\left(c_{g} X_{T}^{i}-\int_{\mathbb{R}} g(x) d \bar{\mu}_{T}^{N, i}(x)\right)^{2}$ is called terminal cost, whilst the term $\left(c_{f} X_{t}^{i}-\int_{\mathbb{R}} f(x) d \bar{\mu}_{t}^{N, i}(x)\right)^{2}$ is called running cost at time $t$. Obviously, the terminal cost becomes smaller as the terminal state $X_{T}^{i}$ of the particle $i$ gets closer to the mean of $g / c_{g}$ over the particles that are connected to $i$; so, the ratio $g / c_{g}$ provides some terminal objective (or terminal target). Similarly, the ratio $f / c_{f}$ provides some running objective. Although this form of interaction may be of a rather restrictive form for modeling, it turns out to be easily tractable from the mathematical point of view. We refer to [7] for a specific instance of application when $f$ and $g$ are linear and $\bar{\mu}_{t}^{N, i}$ is the empirical distribution over all the players (and is thus independent of $i$ ).

For simplicity, we shall set $c_{f}=c_{g}=1$ throughout the text. Regarding the functions $f$ and $g$, we assume them to be smooth enough, but we prefer not to detail this assumption at this stage of the paper and to make it clear when necessary only.

In comparison with models that have been investigated so far, the novel point is the definition of the collection of probability measures $\left(\bar{\mu}_{t}^{N, i}\right)_{0 \leqslant t \leqslant T}$. We call it the collection of empirical marginal distributions of the particles that are connected to $i$. It is defined as

$$
\bar{\mu}_{t}^{N, i}=\frac{1}{N_{i}} \sum_{j=1}^{N} \varepsilon^{i, j} \delta_{X_{t}^{j}}, \quad t \in[0, T]
$$

where $\left(\varepsilon^{i, j}\right)_{1 \leqslant i, j \leqslant N}$ is a collection of bits, that is $\varepsilon^{i, j} \in\{0,1\}$, satisfying the symmetry condition

$$
\varepsilon^{i, j}=\varepsilon^{j, i}, \quad \varepsilon^{i, i}=0 .
$$

In words, the family $\left(\varepsilon^{i, j}\right)_{1 \leqslant i, j \leqslant N}$ forms an undirected graph between the particles: $i$ and $j$ are connected if $\varepsilon^{i, j}=1$; in particular $i$ and $j$ are connected if $j$ and $i$ are connected. Above, $N_{i}$ is 
the number of particles connected to $i$, namely

$$
N_{i}=\sum_{j=1}^{N} \varepsilon^{i, j}
$$

Whenever $N_{i}=0$, we let $\bar{\mu}_{t}^{N, i}$ be the null measure. In such a case this is no longer a probability measure and the action of $\bar{\mu}_{t}^{N, i}$ on $f$ (or $g$ ) is zero.

Throughout the paper, we assume that the $\left(\varepsilon^{i, j}\right)_{1 \leqslant i<j \leqslant N}$ are the realizations of independent and identically distributed Bernoulli random variables of parameter $p \in(0,1)$. With a slight abuse of notation, we still denote by $\left(\varepsilon^{i, j}\right)_{1 \leqslant i<j \leqslant N}$ the corresponding random variables. So, the graph connecting the particles with one another is a realization of an Erdös-Renyi graph. It must be stressed that the collection of random variables $\left(\varepsilon^{i, j}\right)_{1 \leqslant i<j \leqslant N}$ is independent of $\left(X_{0},\left(W_{t}^{i}\right)_{0 \leqslant t \leqslant T}\right)$. To make it clear, we define it on another probability space $(\Xi, \mathcal{G}, \mathbf{P})$. In particular, the realization of the graph is completely frozen in the computation of the costs $\left(J^{i}\right)_{i=1, \cdots, N}$.

\subsection{Notion of equilibria}

Of course, each player is willing to minimize her/his own energy. This prompts us to introduce the notion of Nash equilibrium. As mentioned in introduction, several forms of it are conceivable. Here, we just make use of the simplest version:

Definition 1. A tuple $\left(\left(\alpha_{t}^{i}\right)_{0 \leqslant t \leqslant T}\right)_{i=1, \cdots, N}$ is said to be a Nash equilibrium over open loop controls if, for any $i \in\{1, \cdots, N\}$, for any other adapted and square-integrable control $\left(\beta_{t}\right)_{0 \leqslant t \leqslant T}$, it holds that

$$
J^{i}\left(\left(\alpha_{t}^{1}, \cdots, \alpha_{t}^{i-1}, \beta_{t}, \alpha_{t}^{i+1}, \cdots, \alpha_{t}^{N}\right)_{0 \leqslant t \leqslant T}\right) \geqslant J^{i}\left(\left(\alpha_{t}^{1}, \cdots, \alpha_{t}^{N}\right)_{0 \leqslant t \leqslant T}\right) .
$$

In short, a Nash equilibrium is a consensus between the players: there is no incentive for any particle to leave the consensus. In the above definition, it is said to be over open loop controls since the other players are blind to the change of $\alpha^{i}$ into $\beta$ : In both cases, the other players implement, for any realization of the noise, the same realization of their own control.

In comparison with, Nash equilibria over closed loop controls (as we alluded to in Introduction) would be taken over controls of the form $\left(\alpha_{t}^{i}=\phi^{i}\left(t, X_{t}^{1}, \cdots, X_{t}^{N}\right)\right)_{0 \leqslant t \leqslant T}$ for a feedback function $\phi^{i}$ from $[0, T] \times \mathbb{R}^{N}$ to $\mathbb{R}$. In the latter case, the dynamics for $\left(X_{t}^{1}, \cdots, X_{t}^{N}\right)_{0 \leqslant t \leqslant T}$ become a stochastic differential equation. Under appropriate assumptions on the feedback functions, it is well-solvable. If so, we have the same definition as above for a Nash equilibrium except that, therein, we have to fix the feedback function $\phi^{i}$ instead of the trajectory $\left(\alpha_{t}^{i}\right)_{0 \leqslant t \leqslant T}$. This makes a difference: In words, this means that any other player $j \neq i$ may be willing to take into account the new state of the deviating player $i$ to update her/his control; indeed, while she/he keeps using the same function $\phi^{j}$, she/he implements it at a different tuple. Although it sounds pretty much realistic, we feel better not to address this case in this note.

\subsection{Asymptotic formulation}

We now introduce the asymptotic formulation. The guess is that there should be sufficiently many edges connecting $i$ to the rest of the population to do as if the graph was complete. So, 
we expect the asymptotic game to fit a standard mean field game. The solutions of this latter one are given by the following two step procedure:

First Step. For a fixed flow of probability measures $\left(\mu_{t}\right)_{0 \leqslant t \leqslant T}$, solve the standard optimization problem

$$
\inf _{\left(\alpha_{t}\right)_{0 \leqslant t \leqslant T}} J\left(\left(\alpha_{t}\right)_{0 \leqslant t \leqslant T}\right),
$$

where $\left(\alpha_{t}\right)_{0 \leqslant t \leqslant T}$ is an adapted process with respect to the filtration generated by $\left(X_{0},\left(W_{t}\right)_{0 \leqslant t \leqslant T}\right)$, for $\left(X_{0},\left(W_{t}\right)_{0 \leqslant t \leqslant T}\right) \sim\left(X_{0}^{1},\left(W_{t}^{1}\right)_{0 \leqslant t \leqslant T}\right)$. Above the energy functional is given by

$$
\begin{aligned}
J\left(\left(\alpha_{t}\right)_{0 \leqslant t \leqslant T}\right)=\mathbb{E} & {\left[\frac{1}{2}\left(X_{T}-\int_{\mathbb{R}} g(x) d \mu_{T}\right)^{2}\right.} \\
& \left.+\int_{0}^{T}\left[\frac{1}{2}\left(X_{t}-\int_{\mathbb{R}} f(x) d \mu_{t}\right)^{2}+\frac{1}{2}\left(\alpha_{t}\right)^{2}\right] d t\right],
\end{aligned}
$$

where $\left(X_{t}\right)_{0 \leqslant t \leqslant T}$ solves

$$
X_{t}=X_{0}+\int_{0}^{t} \alpha_{s} d s+W_{t}, \quad t \in[0, T] .
$$

Second Step. Find $\left(\mu_{t}\right)_{0 \leqslant t \leqslant T}$ such that the flow of marginal laws of the optimal process in the first step is $\left(\mu_{t}\right)_{0 \leqslant t \leqslant T}$ itself.

The reader will easily understand that, with our form of interaction, the limiting optimization problem in the first step is linear-quadratic, that is the dynamics of the player are linear in $\alpha$ and the cost functional is quadratic in $(x, \alpha)$. In this regard, the solution is very simple and is known to be, conditional on the realization of the initial condition, a Gaussian process. This is one of the main rationale for the form of the toy model addressed in this note.

Notice also that the asymptotic problem would be of the same form if we used Nash equilibria over closed loop controls.

\section{MASter Equation}

This section is devoted to the analysis of the mean field game.

\subsection{Solvability of the mean field game}

As a starting point, we need a tractable characterization of the solutions of the mean field game formulated in Subsection 2.4. To do so, we make use of the probabilistic approach initiated in [4]. Basically, this approach consists in applying first the Pontryagin principle in order to describe the solution of the standard optimization problem in environment $\left(\mu_{t}\right)_{0 \leqslant t \leqslant T}$, as addressed in the first step right above: The optimal trajectory is described as the forward component of a forward-backward system of two coupled stochastic differential equations, the coefficients of which depend upon $\left(\mu_{t}\right)_{0 \leqslant t \leqslant T}$. Once this forward-backward system has been written down, the point is to implement the fixed point condition stated in the second step above: To do so, we need to replace $\mu_{t}$ in the coefficients by the marginal law at time $t$ of the forward component of the forward-backward system. This leads to a forward-backward 
stochastic differential equation of the McKean-Vlasov type, which characterizes the solutions of the mean field game. It has the form:

$$
\begin{aligned}
& d X_{t}=-Y_{t} d t+d W_{t}, \\
& d Y_{t}=-\left(X_{t}-\mathbb{E}\left[f\left(X_{t}\right)\right]\right) d t+Z_{t} d W_{t}, \quad t \in[0, T], \\
& Y_{T}=X_{T}-\mathbb{E}\left[g\left(X_{T}\right)\right] .
\end{aligned}
$$

Here we assume as before that the initial condition $X_{0}$ of the forward component has been prescribed. It is assumed to be $\mathcal{F}_{0}$ measurable and $\left(W_{t}\right)_{0 \leqslant t \leqslant T}$ is assumed to be an $\left(\mathcal{F}_{t}\right)_{0 \leqslant t \leqslant T^{-}}$ Brownian motion with values in $\mathbb{R}$, for a filtration $\left(\mathcal{F}_{t}\right)_{0 \leqslant t \leqslant T}$ that may no longer coincide with the one described in the previous section. Here, the typical example for $\left(\mathcal{F}_{t}\right)_{0 \leqslant t \leqslant T}$ is the filtration generated by $\mathcal{F}_{0}$ and $\left(W_{t}\right)_{0 \leqslant t \leqslant T}$. Recall also that the solution of the backward equation is the pair $\left(Y_{t}, Z_{t}\right)_{0 \leqslant t \leqslant T}$, which is also required to be adapted. Basically, the martingale integrand $\left(Z_{t}\right)_{0 \leqslant t \leqslant T}$ is used to ensure the adaptedness. It is assumed to be square-integrable.

We now make use of the very simple form of the system (1). By the change of variable $\left(\tilde{Y}_{t}=Y_{t}-X_{t}, \tilde{Z}_{t}=Z_{t}-1\right)_{0 \leqslant t \leqslant T}$, it may be rewritten as

$$
\begin{aligned}
& d X_{t}=-\left(X_{t}+\tilde{Y}_{t}\right) d t+d W_{t}, \\
& d \tilde{Y}_{t}=\left(\tilde{Y}_{t}+\mathbb{E}\left[f\left(X_{t}\right)\right]\right) d t+\tilde{Z}_{t} d W_{t}, \quad t \in[0, T], \\
& \tilde{Y}_{T}=-\mathbb{E}\left[g\left(X_{T}\right)\right] .
\end{aligned}
$$

It is then well checked that $\left(\tilde{Y}_{t}\right)_{0 \leqslant t \leqslant T}$ is necessarily deterministic. Indeed, when $\left(X_{t}\right)_{0 \leqslant t \leqslant T}$ is given, the two last lines form a backward stochastic differential equation. It is known to be uniquely solvable. A particular solution (and hence the solution) is obtained by letting $\left(\tilde{Z}_{t}=0\right)_{0 \leqslant t \leqslant T}$ and by choosing $\left(\tilde{Y}_{t}\right)_{0 \leqslant t \leqslant T}$ as the solution of the ordinary differential equation $[d / d t]\left(\tilde{Y}_{t}\right)=\tilde{Y}_{t}+\mathbb{E}\left[f\left(X_{t}\right)\right]$, with the boundary condition $\tilde{Y}_{T}=-\mathbb{E}\left[g\left(X_{T}\right)\right]$.

Henceforth, any solution to the above forward-backward system must satisfy

$$
\frac{d}{d t}\left(e^{t} \mathbb{E}\left(X_{t}\right)\right)=-e^{t} \tilde{Y}_{t}
$$

which permits to express the forward component as

$$
e^{t} X_{t}=X_{0}-\mathbb{E}\left(X_{0}\right)+e^{t} \mathbb{E}\left(X_{t}\right)+e^{t} \Gamma_{t},
$$

where $\left(\Gamma_{t}\right)_{0 \leqslant t \leqslant T}$ is the Ornstein-Uhlenbeck process

$$
\Gamma_{t}=e^{-t} \int_{0}^{t} e^{s} d W_{s}, \quad t \in[0, T]
$$

Finally, the McKean Vlasov forward backward SDE takes the form:

$$
\begin{aligned}
& d X_{t}=-\left(X_{t}+\tilde{Y}_{t}\right) d t+d W_{t}, \\
& d \tilde{Y}_{t}=\left(\tilde{Y}_{t}+\mathbb{E}\left[f\left(e^{-t}\left(X_{0}-\mathbb{E}\left(X_{0}\right)\right)+\mathbb{E}\left(X_{t}\right)+\Gamma_{t}\right)\right]\right) d t, \quad t \in[0, T], \\
& \tilde{Y}_{T}=-\mathbb{E}\left[g\left(e^{-T}\left(X_{0}-\mathbb{E}\left(X_{0}\right)\right)+\mathbb{E}\left(X_{T}\right)+\Gamma_{T}\right)\right] .
\end{aligned}
$$


Taking the mean and recalling (2), we deduce the following proposition, the proof of which is straightforward.

Proposition 1. The McKean Vlasov forward backward SDE (1) is uniquely solvable if and only if the following deterministic system is uniquely solvable:

$$
\begin{aligned}
& d m_{t}=-\left(m_{t}+\tilde{Y}_{t}\right) d t \\
& d \tilde{Y}_{t}=\left(\tilde{Y}_{t}+\mathbb{E}\left[f\left(e^{-t}\left(X_{0}-\mathbb{E}\left(X_{0}\right)\right)+m_{t}+\Gamma_{t}\right)\right]\right) d t, \quad t \in[0, T], \\
& \tilde{Y}_{T}=-\mathbb{E}\left[g\left(e^{-T}\left(X_{0}-\mathbb{E}\left(X_{0}\right)\right)+m_{T}+\Gamma_{T}\right)\right],
\end{aligned}
$$

with $m_{0}=\mathbb{E}\left(X_{0}\right)$ as initial condition, in which case $\mathbb{E}\left(X_{t}\right)=m_{t}$, for all $t \in[0, T]$.

By the new change of variable $\left(\bar{m}_{t}=e^{t} m_{t}-\mathbb{E}\left(X_{0}\right), \bar{Y}_{t}=e^{-t} \tilde{Y}_{t}\right)_{0 \leqslant t \leqslant T}$, the above system is equivalent to

$$
\begin{aligned}
& d \bar{m}_{t}=-e^{2 t} \bar{Y}_{t} d t, \quad \bar{m}_{0}=0, \\
& d \bar{Y}_{t}=e^{-t} \mathbb{E}\left[f\left(e^{-t}\left(X_{0}+\bar{m}_{t}\right)+\Gamma_{t}\right)\right] d t, \\
& \bar{Y}_{T}=-e^{-T} \mathbb{E}\left[g\left(e^{-T}\left(X_{0}+\bar{m}_{T}\right)+\Gamma_{T}\right)\right],
\end{aligned}
$$

in which case $\mathbb{E}\left(X_{t}\right)=e^{-t}\left(\bar{m}_{t}+\mathbb{E}\left(X_{0}\right)\right)$. The following proposition provides conditions under which existence and uniqueness hold true.

Proposition 2. Assume that $f$ and $g$ are non-increasing and Lipchitz-continuous functions. Then, for any square-integrable initial condition $X_{0}$, there exists a unique solution to the system (3).

Proof. The result follows from a standard fact in the theory of forward-backward systems. It suffices to observe that, when $X_{0}$ is given, the functions

$$
\begin{aligned}
& \mathbb{R} \ni x \mapsto-\mathbb{E}\left[f\left(e^{-t}\left(X_{0}+x\right)+\Gamma_{t}\right)\right], \quad t \in[0, T], \\
& \mathbb{R} \ni x \mapsto-\mathbb{E}\left[g\left(e^{-T}\left(X_{0}+x\right)+\Gamma_{T}\right)\right],
\end{aligned}
$$

are non-decreasing and Lipschitz continuous. This permits to regard the system (3) as a deterministic forward-backward system with non-decreasing and Lipschitz continuous coefficients. It is then known to be uniquely solvable, see for instance [20].

Actually, we can even prove a more general stability result.

Proposition 3. Assume that $f$ and $g$ satisfy the same assumptions as above. Then, there exists a constant $C$ such that, for any two initial conditions $X_{0}$ and $X_{0}^{\prime}$ of the system (1),

$$
\left|\bar{Y}_{0}-\bar{Y}_{0}^{\prime}\right| \leqslant C \mathbb{E}\left[\left|X_{0}-X_{0}^{\prime}\right|\right]
$$

where $\left(\bar{m}_{t}, \bar{Y}_{t}\right)_{0 \leqslant t \leqslant T}$ and $\left(\bar{m}_{t}^{\prime}, \bar{Y}_{t}^{\prime}\right)_{0 \leqslant t \leqslant T}$ are the corresponding solutions to $(3)$. 
Proof. Again, the proof is pretty standard. It consists in regarding the product $\left(\left(\bar{m}_{t}^{\prime}-\bar{m}_{t}\right)\left(\bar{Y}_{t}^{\prime}-\right.\right.$ $\left.\left.\bar{Y}_{t}\right)\right)_{0 \leqslant t \leqslant T}$. We compute

$$
\begin{aligned}
& \frac{d}{d t}\left[\left(\bar{m}_{t}^{\prime}-\bar{m}_{t}\right)\left(\bar{Y}_{t}^{\prime}-\bar{Y}_{t}\right)\right] \\
& =-e^{2 t}\left(\tilde{Y}_{t}^{\prime}-\tilde{Y}_{t}\right)^{2} \\
& \quad+e^{-t}\left(\bar{m}_{t}^{\prime}-\bar{m}_{t}\right)\left(\mathbb{E}\left[f\left(e^{-t}\left(X_{0}^{\prime}+\bar{m}_{t}^{\prime}\right)+\Gamma_{t}\right)\right]-\mathbb{E}\left[f\left(e^{-t}\left(X_{0}+\bar{m}_{t}\right)+\Gamma_{t}\right)\right]\right) .
\end{aligned}
$$

Since $f$ is Lipchitz-continuous and non-increasing, we can find a constant $C$, only depending on the coefficients, such that

$$
\begin{aligned}
\frac{d}{d t}\left[\left(\bar{m}_{t}^{\prime}-\bar{m}_{t}\right)\left(\bar{Y}_{t}^{\prime}-\bar{Y}_{t}\right)\right] \leqslant & -e^{2 t}\left(\bar{Y}_{t}^{\prime}-\bar{Y}_{t}\right)^{2} \\
& +C\left|\bar{m}_{t}^{\prime}-\bar{m}_{t}\right| \mathbb{E}\left[\left|X_{0}^{\prime}-X_{0}\right|\right]
\end{aligned}
$$

which yields

$$
\begin{aligned}
& -\left(\bar{m}_{t}^{\prime}-\bar{m}_{t}\right)\left(\bar{Y}_{t}^{\prime}-\bar{Y}_{t}\right)+\int_{t}^{T} e^{2 s}\left(\bar{Y}_{s}^{\prime}-\bar{Y}_{s}\right)^{2} d s \\
& \leqslant-\left(\bar{m}_{T}^{\prime}-\bar{m}_{T}\right)\left(\bar{Y}_{T}^{\prime}-\bar{Y}_{T}\right)+C \mathbb{E}\left[\left|X_{0}^{\prime}-X_{0}\right|\right] \int_{t}^{T}\left|\bar{m}_{s}^{\prime}-\bar{m}_{s}\right| d s .
\end{aligned}
$$

Now, using the fact that $g$ is Lipschitz-continuous and non-increasing,

$$
\begin{aligned}
& -\left(\bar{m}_{T}^{\prime}-\bar{m}_{T}\right)\left(\bar{Y}_{T}^{\prime}-\bar{Y}_{T}\right) \\
& =\left(\bar{m}_{T}^{\prime}-\bar{m}_{T}\right) e^{-T}\left(\mathbb{E}\left[g\left(e^{-T}\left(X_{0}^{\prime}+\bar{m}_{T}^{\prime}\right)+\Gamma_{T}\right)\right]\right. \\
& \leqslant C\left|m_{T}^{\prime}-\bar{m}_{T}\right| \mathbb{E}\left[\left|X_{0}^{\prime}-X_{0}\right|\right] .
\end{aligned}
$$

Recalling that $\bar{m}_{0}=\bar{m}_{0}^{\prime}=0$, we end up with

$$
\begin{aligned}
& \int_{0}^{T} e^{2 s}\left(\bar{Y}_{s}^{\prime}-\bar{Y}_{s}\right)^{2} d s \\
& \leqslant C\left|m_{T}^{\prime}-\bar{m}_{T}\right| \mathbb{E}\left[\left|X_{0}^{\prime}-X_{0}\right|\right]+C \mathbb{E}\left[\left|X_{0}^{\prime}-X_{0}\right|\right] \int_{t}^{T}\left|\bar{m}_{s}^{\prime}-\bar{m}_{s}\right| d s .
\end{aligned}
$$

Returning to the forward equation, we deduce that

$$
\begin{aligned}
& \sup _{0 \leqslant t \leqslant T}\left|\bar{m}_{t}-\bar{m}_{t}^{\prime}\right|^{2} \\
& \quad \leqslant C\left|m_{T}^{\prime}-\bar{m}_{T}\right| \mathbb{E}\left[\left|X_{0}^{\prime}-X_{0}\right|\right]+C \mathbb{E}\left[\left|X_{0}^{\prime}-X_{0}\right|\right] \int_{t}^{T}\left|\bar{m}_{s}^{\prime}-\bar{m}_{s}\right| d s .
\end{aligned}
$$


By a standard convexity inequality, we obtain

$$
\sup _{0 \leqslant t \leqslant T}\left|\bar{m}_{t}-\bar{m}_{t}^{\prime}\right| \leqslant C \mathbb{E}\left[\left|X_{0}^{\prime}-X_{0}\right|\right]
$$

Plugging into the backward equation, we easily complete the proof.

\subsection{Master field}

The combination of Propositions 2 and 3 permits to define a Lipschitz continuous mapping

$$
L^{2}\left(\Omega, \mathcal{F}_{0}, \mathbb{P} ; \mathbb{R}\right) \ni X_{0} \mapsto \bar{Y}_{0} \in \mathbb{R}
$$

which maps the initial condition of the system (1) onto the initial value of the backward component of the system (3). Remarkably, the Lipschitz property holds true with respect to the $L^{1}$ norm. Importantly, as the coefficients of (3) only depend on $X_{0}$ through its distribution, uniqueness of the solution to (3) says that the same holds true for $\bar{Y}_{0}$ : it only depends on $X_{0}$ through its distribution. So, denoting by $\mathcal{P}_{2}(\mathbb{R})$ the space of probability measures on $\mathbb{R}$ with a finite second-order moment, the above mapping may be regarded as a mere mapping from $\mathcal{P}_{2}(\mathbb{R})$ into $\mathbb{R}$, namely

$$
\mathcal{V}(0, \cdot): \mathcal{P}_{2}(\mathbb{R}) \ni \mu_{0} \mapsto \bar{Y}_{0} \in \mathbb{R}
$$

where the right-hand side is computed for some $X_{0} \sim \mu_{0}$. This mapping is Lipschitz continuous for the 1-Wasserstein distance $\mathcal{W}_{1}$ (and so for the 2-Wasserstein distance $\mathcal{W}_{2}$ as well since $\mathcal{W}_{1}$ is dominated by $\mathcal{W}_{2}$ ).

Of course, we can easily extend the argument to any initial time $t_{0} \in[0, T]$ by initializing the system (1) at time $t_{0}$. In words, this amounts to initialize the system (3) at time $t_{0}$ or, equivalently, in shifting it to $\left[0, T-t_{0}\right]$. The mapping $\mathcal{V}\left(t_{0}, \cdot\right)$ is then defined as

$$
\mathcal{V}\left(t_{0}, \cdot\right): \mathcal{P}_{2}(\mathbb{R}) \ni \mu_{0} \mapsto \bar{Y}_{t_{0}} \in \mathbb{R}
$$

where the right-hand side is the solution of the backward equation in (3), when this latter one is set on $\left[0, T-t_{0}\right]$ instead of $[0, T], e^{T}$ and $\Gamma_{T}$ in the terminal condition being replaced by $e^{T-t_{0}}$ and $\Gamma_{T-t_{0}}$, and $X_{0}$ being some $\mathcal{F}_{0}$-measurable random variable with the same distribution as the initial condition at time $t_{0}$ of the system (1).

A crucial fact for the analysis of the finite player game equilibria is to prove that the mapping $\mathcal{V}$ is smooth with respect to the measure argument, or, say to simplify, that the mapping $\mathcal{V}(0, \cdot)$ is smooth. This requires to give first a meaning to the underlying notion of derivative. To do so, we shall use Lions' approach to the so-called Wasserstein derivative, as introduced in [17], see also $[2,5]$. This requires to define first the lifting of $\mathcal{V}(0, \cdot)$ to $L^{2}\left(\Omega, \mathcal{F}_{0}, \mathbb{P} ; \mathbb{R}\right)$, namely

$$
\hat{\mathcal{V}}\left(0, X_{0}\right)=\mathcal{V}\left(0, \mathcal{L}\left(X_{0}\right)\right)
$$

where $\mathcal{L}\left(X_{0}\right)$ is the law of $X_{0}$. We then say that $\mathcal{V}(0, \cdot)$ is continuously differentiable if the lift $\hat{\mathcal{V}}(0, \cdot)$ is Fréchet continuously differentiable. Of course, this makes sense as long as the space $\left(\Omega, \mathcal{F}_{0}, \mathbb{P}\right)$ is rich enough so that, for any $\mu_{0} \in \mathcal{P}_{2}(\mathbb{R})$, there exists a random variable $X_{0} \in L^{2}\left(\Omega, \mathcal{F}_{0}, \mathbb{P} ; \mathbb{R}\right)$ such that $X_{0} \sim \mu_{0}$, which we can assume to be true without any loss of generality. A crucial point with Lions' approach to Wasserstein differential calculus is that the 
Fréchet derivative of $\hat{\mathcal{V}}(0, \cdot)$, which can be identified with a square-integrable random variable, may be represented at point $X_{0}$ as $\partial_{\mu} \mathcal{V}\left(0, \mu_{0}\right)\left(X_{0}\right)$ for a mapping $\partial_{\mu} \mathcal{V}\left(0, \mu_{0}\right)(\cdot): \mathbb{R} \ni v \mapsto$ $\partial_{\mu} \mathcal{V}\left(0, \mu_{0}\right)(v) \in \mathbb{R}$. This latter function plays the role of Wasserstein derivative of $\mathcal{V}(0, \cdot)$ in the measure argument.

Second-order derivatives may be defined accordingly. The function $\mathcal{V}(0, \cdot)$ is said to be twice continuously differentiable if we can find, for each $\mu \in \mathcal{P}_{2}(\mathbb{R})$, a version of $\mathbb{R} \ni v \mapsto \partial_{\mu} \mathcal{V}(0, \mu)(v)$ (which is a priori defined as a function in $L^{2}(\mathbb{R}, \mu ; \mathbb{R})$ ) such that $\mathcal{P}_{2}(\mathbb{R}) \times \mathbb{R} \ni(\mu, v) \mapsto$ $\partial_{\mu} \mathcal{V}(0, \mu)(v)$ is continuous, differentiable in $v$ when $\mu$ is fixed, and differentiable in $\mu$ when $v$ is fixed, with jointly continuous derivatives, namely $\mathcal{P}_{2}(\mathbb{R}) \times \mathbb{R} \ni(\mu, v) \mapsto \partial_{v} \partial_{\mu} \mathcal{V}(0, \mu)(v)$ is continuous and $\mathcal{P}_{2}(\mathbb{R}) \times \mathbb{R} \times \mathbb{R} \ni\left(\mu, v, v^{\prime}\right) \mapsto \partial_{\mu}^{2} \mathcal{V}(0, \mu)\left(v, v^{\prime}\right)$ is continuous (here the variable $v^{\prime}$ accounts for the second-order derivative in the direction $\mu$ ).

\subsection{Proving the smoothness of $\mathcal{V}$}

Generally speaking, the smoothness of $\mathcal{V}$ at order two (which is the one we need for writing down the master equation, see Subsection 3.4) is addressed in the two papers [3, 8] and in the monograph [6]. The general method consists in proving first that $\mathcal{V}(t, \cdot)$ is a smooth function of the measure argument for $t$ close to $T$, or equivalently, in establishing the smoothness of $\mathcal{V}(t, \cdot)$ for $t \in[0, T]$ when $T$ is less than some small $\delta>0$. In order to do so, $f$ and $g$ must be smooth enough, say bounded and three times differentiable, with bounded derivatives. The idea is then to show that the solution to $(3)$ varies smoothly with $X_{0} \in L^{2}\left(\Omega, \mathcal{F}_{0}, \mathbb{P} ; \mathbb{R}\right)$ when $T \leqslant \delta$; this approach should be regarded as a flow method, but with an initial condition in a space of infinite dimension. In this regard, the condition $T \leqslant \delta$ plays a key role, as it permits to define the successive derivatives of the flow as the solutions of differentiated forward-backward systems. We feel better not to detail the analysis and to refer the reader to the aforementioned citations. The crux is then to pass from a small time horizon $T$ to an arbitrary time horizon. This is the point where the Lipschitz control of $\mathcal{V}$ comes in. In [6] and [8], it is shown that, for a given $T>0$, the analysis of the smoothness of $\mathcal{V}$ could be extended from the neighborhood $[T-\delta, T]$ of the terminal time $T$ to the whole $[0, T]$ by iterating backwards the small time analysis along a sequence of small intervals of the form $[T-(n+1) \delta, T-n \delta]$, with $n$ such that $T-n \delta>0$. Importantly, $\delta$ can be assumed to be the same throughout the induction. To make it clear, it must be stressed that the time length $\delta$, as defined right above, depends in fact on the Lipschitz constants of $f$ and $g$ with respect to the law of $X_{0}$ for the distance $\mathcal{W}_{1}$, with the slight abuse that $f$ and $g$ are seen as functions of the law of $X_{0}$. When iterating the small time analysis as we just described, the terminal boundary condition has to be updated on any new interval, namely $g$ has to be replaced by $\mathcal{V}(T-n \delta, \cdot)$ when working on the interval $[T-(n+1) \delta, T-n \delta]$.

For the sake of illustration, we provide an explicit example.

Proposition 4. Assume that $f$ is null and that $g$ is non-increasing, bounded and smooth, with bounded derivatives. Then, the function $\mathcal{V}$ is jointly continuous. It is differentiable in time and $[\partial \mathcal{V} / \partial t]$ is jointly continuous. Also, for any $t \in[0, T]$, the function $\mathcal{V}(t, \cdot)$ is differentiable with respect to the measure argument and the function

$$
[0, T] \times \mathcal{P}_{2}(\mathbb{R}) \times \mathbb{R} \ni(t, \mu, v) \mapsto \partial_{\mu} \mathcal{V}(t, \mu)(v)
$$


is bounded and continuous. It is differentiable in the variable $v$ and in the variable $\mu$ and the functions

$$
\begin{aligned}
& {[0, T] \times \mathcal{P}_{2}(\mathbb{R}) \times \mathbb{R} \ni(t, \mu, v) \mapsto \partial_{v} \partial_{\mu} \mathcal{V}(t, \mu)(v)} \\
& {[0, T] \times \mathcal{P}_{2}(\mathbb{R}) \times \mathbb{R} \times \mathbb{R} \ni\left(t, \mu, v, v^{\prime}\right) \mapsto \partial_{\mu}^{2} \mathcal{V}(t, \mu)\left(v, v^{\prime}\right)}
\end{aligned}
$$

are bounded and continuous.

Remark 1. As a corollary of the proof given below, we obtain the following representation for $\mathcal{V}(0, \mu)$ (when $f$ is null):

$$
\mathcal{V}(0, \mu)=-\frac{e^{-T}}{\sinh (T)} \bar{m}_{T},
$$

where $\bar{m}_{T}$ is the unique fixed point of the equation $\bar{m}_{T}=\sinh (T) \mathbb{E}\left[g\left(e^{-T}\left(X_{0}+\bar{m}_{T}\right)+\Gamma_{T}\right)\right]$, where $X_{0} \sim \mu$ is independent of $\Gamma_{T}$.

Proof. If $f=0$, the solution of the forward-backward system (3) is given by the fixed point condition

$$
\begin{aligned}
\bar{m}_{T} & =\int_{0}^{T} e^{2 t-T} \mathbb{E}\left[g\left(e^{-T}\left(X_{0}+\bar{m}_{T}\right)+\Gamma_{T}\right)\right] d t \\
& =\sinh (T) \mathbb{E}\left[g\left(e^{-T}\left(X_{0}+\bar{m}_{T}\right)+\Gamma_{T}\right)\right] .
\end{aligned}
$$

In order to compute the derivative of $\mathcal{V}(0, \cdot)$, we proceed as follows. We consider two random variables $X_{0}$ and $Y$ in $L^{2}\left(\Omega, \mathcal{F}_{0}, \mathbb{P} ; \mathbb{R}\right)$, with $\mathbb{E}\left[|Y|^{2}\right] \leqslant 1$. For $\varepsilon \in \mathbb{R}$, we call $\left(\bar{m}_{t}^{\varepsilon}, \bar{Y}_{t}^{\varepsilon}\right)_{0 \leqslant t \leqslant T}$ the solution to (3) when (1) is initialized with $X_{0}+\varepsilon Y$. Since $\left(\bar{Y}_{t}^{\varepsilon}\right)_{0 \leqslant t \leqslant T}$ is constant in time,

$$
\bar{m}_{T}^{\varepsilon}=-\frac{1}{2}\left(e^{2 T}-1\right) \mathcal{V}\left(0, \mathcal{L}\left(X_{0}+\varepsilon Y\right)\right) .
$$

By Proposition 3,

$$
\left|\bar{m}_{T}^{\varepsilon}-\bar{m}_{T}^{0}\right| \leqslant C \varepsilon \mathbb{E}[|Y|] .
$$

Letting $e_{T}=e^{-T} \sinh (T)$, we have

$$
\begin{aligned}
\bar{m}_{T}^{\varepsilon}= & \sinh (T) \mathbb{E}\left[g\left(e^{-T}\left(X_{0}+\varepsilon Y+\bar{m}_{T}^{\varepsilon}\right)+\Gamma_{T}\right)\right] \\
= & \sinh (T) \mathbb{E}\left[g\left(e^{-T}\left(X_{0}+\bar{m}_{T}^{0}+\varepsilon Y+\left(\bar{m}_{T}^{\varepsilon}-\bar{m}_{T}^{0}\right)\right)+\Gamma_{T}\right)\right] \\
= & \sinh (T) \mathbb{E}\left[g\left(e^{-T}\left(X_{0}+\bar{m}_{T}^{0}\right)+\Gamma_{T}\right)\right] \\
& +\varepsilon e_{T} \mathbb{E}\left[g^{\prime}\left(e^{-T}\left(X_{0}+\bar{m}_{T}^{0}\right)+\Gamma_{T}\right) Y\right] \\
& +e_{T} \mathbb{E}\left[g^{\prime}\left(e^{-T}\left(X_{0}+\bar{m}_{T}^{0}\right)+\Gamma_{T}\right)\right]\left(\bar{m}_{T}^{\varepsilon}-\bar{m}_{T}^{0}\right) \\
& +O\left(\varepsilon^{2}\right)
\end{aligned}
$$

where the Landau symbol $O\left(\varepsilon^{2}\right)$ satisfies $\left|O\left(\varepsilon^{2}\right)\right| \leqslant C \varepsilon^{2}$ for a constant $C$ independent of $X_{0}$ and $Y$. Recalling that $\sinh (T) \mathbb{E}\left[g\left(e^{-T}\left(X_{0}+\bar{m}_{T}^{0}\right)+\Gamma_{T}\right)\right]=\bar{m}_{T}^{0}$, we get

$$
\begin{array}{r}
\left(1-e_{T} \mathbb{E}\left[g^{\prime}\left(e^{-T}\left(X_{0}+\bar{m}_{T}^{0}\right)+\Gamma_{T}\right)\right]\right)\left(\bar{m}_{T}^{\varepsilon}-\bar{m}_{T}^{0}\right) \\
=\varepsilon e_{T} \mathbb{E}\left[g^{\prime}\left(e^{-T}\left(X_{0}+\bar{m}_{T}^{0}\right)+\Gamma_{T}\right) Y\right]+O\left(\varepsilon^{2}\right),
\end{array}
$$


that is, since $g^{\prime} \leqslant 0$,

$$
\bar{m}_{T}^{\varepsilon}-\bar{m}_{T}^{0}=\varepsilon e_{T} \frac{\mathbb{E}\left[g^{\prime}\left(e^{-T}\left(X_{0}+\bar{m}_{T}^{0}\right)+\Gamma_{T}\right) Y\right]}{1-e_{T} \mathbb{E}\left[g^{\prime}\left(e^{-T}\left(X_{0}+\bar{m}_{T}^{0}\right)+\Gamma_{T}\right)\right]}+O\left(\varepsilon^{2}\right) .
$$

Recall from (5) that

$$
\mathcal{V}\left(0, \mathcal{L}\left(X_{0}\right)\right)=-e^{-2 T} e_{T}^{-1} \bar{m}_{T}^{0},
$$

which shows that $\mathcal{V}(0, \cdot)$ is differentiable with respect to the measure argument; also, for any $\mu \in \mathcal{P}_{2}(\mathbb{R})$ and $v \in \mathbb{R}$,

$$
\partial_{\mu} \mathcal{V}(0, \mu)(v)=-\frac{e^{-2 T} \mathbb{E}\left[g^{\prime}\left(e^{-T} v-e^{T} e_{T} \mathcal{V}(0, \mu)+\Gamma_{T}\right)\right]}{1-e_{T} \mathbb{E}\left[g^{\prime}\left(e^{-T} X_{0}-e^{T} e_{T} \mathcal{V}(0, \mu)+\Gamma_{T}\right)\right]}
$$

It is absolutely obvious to check that $\partial_{\mu} \mathcal{V}(0, \mu)(v)$ can be differentiated once more with respect to $\mu$ and $v$, namely

$$
\partial_{v} \partial_{\mu} \mathcal{V}(0, \mu)(v)=-\frac{e^{-3 T} \mathbb{E}\left[g^{\prime \prime}\left(e^{-T} v-e^{T} e_{T} \mathcal{V}(0, \mu)+\Gamma_{T}\right)\right]}{1-e_{T} \mathbb{E}\left[g^{\prime}\left(e^{-T} X_{0}-e^{T} e_{T} \mathcal{V}(0, \mu)+\Gamma_{T}\right)\right]},
$$

whilst the chain rule yields

$$
\begin{aligned}
& \partial_{\mu}^{2} \mathcal{V}(0, \mu)\left(v, v^{\prime}\right) \\
& =e^{-T} e_{T} \partial_{\mu} \mathcal{V}(0, \mu)\left(v^{\prime}\right)\left(\frac{\mathbb{E}\left[g^{\prime \prime}\left(e^{-T} v-e^{T} e_{T} \mathcal{V}(0, \mu)+\Gamma_{T}\right)\right]}{1-e_{T} \mathbb{E}\left[g^{\prime}\left(e^{-T} X_{0}-e^{T} e_{T} \mathcal{V}(0, \mu)+\Gamma_{T}\right)\right]}\right. \\
& \left.+\frac{e_{T} \mathbb{E}\left[g^{\prime}\left(e^{-T} v-e^{T} e_{T} \mathcal{V}(0, \mu)+\Gamma_{T}\right)\right] \mathbb{E}\left[g^{\prime \prime}\left(e^{-T} v-e^{T} e_{T} \mathcal{V}(0, \mu)+\Gamma_{T}\right)\right]}{\left(1-e_{T} \mathbb{E}\left[g^{\prime}\left(e^{-T} X_{0}-e^{T} e_{T} \mathcal{V}(0, \mu)+\Gamma_{T}\right)\right]\right)^{2}}\right) .
\end{aligned}
$$

The above formulas can be extended to any time $t \in[0, T]$ (instead of the sole $t=0$ ). It suffices to replace $T$ by $T-t, e_{T}$ by $e_{T-t}, \Gamma_{T}$ by $\Gamma_{T-t}$ and then $\mathcal{V}(0, \mu)$ by $\mathcal{V}(t, \mu)$. By (4) and (5), it holds that

$$
\mathcal{V}(t, \mu)=-e^{-(T-t)} \int_{\mathbb{R}} \mathbb{E}\left[g\left(e^{-(T-t)} v-e^{T-t} e_{T-t} \mathcal{V}(t, \mu)+\Gamma_{T-t}\right)\right] d \mu(v) .
$$

This prompts us to let

$$
\Phi(t, y)=-e^{-(T-t)} \int_{\mathbb{R}} \mathbb{E}\left[g\left(e^{-(T-t)} v-e^{T-t} e_{T-t} y+\Gamma_{T-t}\right)\right] d \mu(v),
$$

for $t \in[0, T]$ and $y \in \mathbb{R}$. Obviously, $\Phi$ is continuously differentiable with respect to $y$ and

$$
\frac{\partial \Phi}{\partial y}(t, y)=e_{T-t} \int_{\mathbb{R}} \mathbb{E}\left[g^{\prime}\left(e^{-(T-t)} v-e^{T-t} e_{T-t} y+\Gamma_{T-t}\right)\right] d \mu(v) \leqslant 0 .
$$


Notice also from Itô's formula that

$$
\begin{aligned}
& \Phi(t, y) \\
& =-e^{-(T-t)}\left(\int_{\mathbb{R}} \mathbb{E}\left[g\left(e^{-(T-t)} v-e^{T-t} e_{T-t} y\right)\right] d \mu(v)\right. \\
& \left.+\frac{1}{2} \int_{0}^{T-t} d s \int_{\mathbb{R}} \mathbb{E}\left[g^{\prime \prime}\left(e^{-(T-t)} v-e^{T-t} e_{T-t} y+e^{s-(T-t)} \Gamma_{s}\right)\right] e^{2(s-(T-t))} d \mu(v)\right),
\end{aligned}
$$

so that $\Phi$ is continuously differentiable with respect to $t$. Observing that

$$
\mathcal{V}(t, \mu)=\Phi(t, \mathcal{V}(t, \mu))
$$

we deduce from the implicit function theorem that, for any $\mu \in \mathcal{P}_{2}(\mathbb{R})$, the function $\mathcal{V}(\cdot, \mu)$ is continuously differentiable. Also, for any $t \in[0, T]$,

$$
\frac{\partial}{\partial t} \mathcal{V}(t, \mu)=\left(1-\frac{\partial \Phi}{\partial y}(t, \mathcal{V}(t, \mu))\right)^{-1} \frac{\partial \Phi}{\partial t}(t, \mathcal{V}(t, \mu))
$$

Since $\mathcal{V}$ is Lipschitz-continuous in the measure argument, we deduce that it is in fact jointly continuous. Also, all the derivatives we computed above are jointly continuous in all their arguments (including time).

\subsection{Form of the master equation}

Returning to (1) (and assuming that $f$ may be non-zero) and recalling that $\left(\tilde{Y}_{t}=e^{t} \bar{Y}_{t}\right)_{0 \leqslant t \leqslant T}$, and that $\left(Y_{t}=X_{t}+\tilde{Y}_{t}\right)_{0 \leqslant t \leqslant T}$, we finally end up with the fact that

$$
Y_{0}=X_{0}+\bar{Y}_{0}=X_{0}+\mathcal{V}\left(0, \mathcal{L}\left(X_{0}\right)\right)
$$

and more generally, by regarding $X_{t}$ as the initial condition when the system is restricted to the interval $[t, T]$, we also have

$$
Y_{t}=X_{t}+\tilde{Y}_{t}=X_{t}+\mathcal{V}\left(t, \mathcal{L}\left(X_{t}\right)\right)
$$

which prompts us to let

$$
\mathcal{U}(t, x, \mu)=x+\mathcal{V}(t, \mu), \quad(t, x, \mu) \in[0, T] \times \mathbb{R} \times \mathcal{P}_{2}(\mathbb{R})
$$

We then claim:

Proposition 5. Assume that $\mathcal{V}$ satisfies the conclusion of Proposition 4 , then the function $\mathcal{U}$ satisfies the $P D E$, set on $[0, T] \times \mathbb{R} \times \mathcal{P}(\mathbb{R})$,

$$
\begin{aligned}
& \partial_{t} \mathcal{U}(t, x, \mu)-\mathcal{U}(t, x, \mu) \partial_{x} \mathcal{U}(t, x, \mu)+\frac{1}{2} \partial_{x}^{2} \mathcal{U}(t, x, \mu) \\
& \quad-\int_{\mathbb{R}} \mathcal{U}(t, v, \mu) \partial_{\mu} \mathcal{U}(t, x, \mu)(v) d \mu(v)+\frac{1}{2} \int_{\mathbb{R}} \partial_{v} \partial_{\mu} \mathcal{U}(t, x, \mu)(v) d \mu(v) \\
& \quad+x-\int_{\mathbb{R}} f(v) d \mu(v)=0
\end{aligned}
$$


for $(t, x, \mu) \in[0, T] \times \mathbb{R} \times \mathcal{P}_{2}(\mathbb{R})$, with the terminal boundary condition

$$
\mathcal{U}(T, x, \mu)=x-\int_{\mathbb{R}} g(v) d \mu(v)
$$

Equation (8) is called the master equation of the system (1). Notice that, because of the special form of $\mathcal{U}$, the partial derivatives of $\mathcal{U}$ in $x$ here simplify.

Proof. The proof of Proposition 5 consists in expanding (6) by means of a suitable version of the chain rule for flows of marginal distributions of a diffusion process, see Lemma 1, and by identifying the expansion with the backward equation in (1).

The following lemma is taken from $[5,8]$.

Lemma 1. Let $\left(\chi_{t}\right)_{0 \leqslant t \leqslant T}$ be a real-valued Itô process of the form:

$$
d \chi_{t}=\beta_{t} d t+d W_{t}
$$

where $\left(\beta_{t}\right)_{0 \leqslant t \leqslant T}$ is a real-valued adapted process satisfying

$$
\mathbb{E} \int_{0}^{T} \beta_{t}^{2} d t<\infty
$$

If $\mathcal{U}:[0, T] \times \mathbb{R} \times \mathcal{P}(\mathbb{R}) \rightarrow \mathbb{R}$ satisfy the assumption of Proposition 5 , then the process $\left(\mathcal{U}\left(t, X_{t}, \mathcal{L}\left(X_{t}\right)\right)\right)_{0 \leqslant t \leqslant T}$ expands as an Itô process, namely

$$
\begin{aligned}
& d\left[\mathcal{U}\left(t, X_{t}, \mathcal{L}\left(X_{t}\right)\right)\right] \\
& =\left(\partial_{t} \mathcal{U}\left(t, X_{t}, \mathcal{L}\left(X_{t}\right)\right)+\partial_{x} \mathcal{U}\left(t, X_{t}, \mathcal{L}\left(X_{t}\right)\right) \beta_{t}+\frac{1}{2} \partial_{x}^{2} \mathcal{U}\left(t, X_{t}, \mathcal{L}\left(X_{t}\right)\right)\right. \\
& \left.\quad+\mathbb{E}\left[\partial_{\mu} \mathcal{U}\left(t, x, \mathcal{L}\left(X_{t}\right)\right)\left(X_{t}\right) \beta_{t}\right]_{x=X_{t}}+\frac{1}{2} \mathbb{E}\left[\partial_{v} \partial_{\mu} \mathcal{U}\left(t, x, \mathcal{L}\left(X_{t}\right)\right)\left(X_{t}\right)\right]_{x=X_{t}}\right) d t \\
& \quad+\partial_{x} \mathcal{U}\left(t, X_{t}, \mathcal{L}\left(X_{t}\right)\right) d W_{t},
\end{aligned}
$$

for $t \in[0, T]$.

\section{Convergence of the Finite Games}

We now address the validity of the limiting formulation. To do so, we focus on the case when the equilibria in the finite player game are taken over open loop controls, see Definition 1.

Throughout this section, we assume that the conclusion of Proposition 4 holds true.

\subsection{First-order condition}

Actually, we are going to simplify once more the problem. Instead of addressing the convergence of the equilibria, we shall address the convergence of the solutions to the first-order condition that any equilibrium (if it exists) must satisfy. Equivalently, this amounts to say that we shall not address the existence of the equilibria. To make it clear, recall indeed that an equilibrium is defined through a collection of $N$ partial minimization problems, see Definition 
1. As usual with minimization problems, we may provide, by expanding at the first-order the cost functional around the equilibrium, a necessary but possibly not sufficient condition that any equilibrium must satisfy. This is what we call a first-order condition: Somehow, it is the analogue of the standard condition stating that the minimum of a function $f$ of the real variable must be a zero of the derivative of $f$ (which is indeed known to be a necessary but possibly not sufficient condition).

We use the same setting as in Section 2. Namely, we are given a collection of $N$ real-valued Brownian motions $\left(\left(W_{t}^{i}\right)_{0 \leqslant t \leqslant T}\right)_{i=1, \cdots, N}$, where $T$ is as before and $N$ is a fixed integer. These processes are constructed on a probability space $(\Omega, \mathcal{F}, \mathbb{P})$ and the filtration generated by the noises and possibly augmented with an initial $\sigma$-field $\mathcal{F}_{0}$, independent of $\left(\left(W_{t}^{i}\right)_{0 \leqslant t \leqslant T}\right)_{i=1, \cdots, N}$, is denoted by $\left(\mathcal{F}_{t}\right)_{0 \leqslant t \leqslant T}$. Also, the initial conditions $\left(X_{0}^{i}\right)_{i=1, \cdots, N}$ are assumed to be $\mathcal{F}_{0}$-measurable, square-integrable, independent and identically distributed.

In game theory, the aforementioned first-order condition that any equilibrium must satisfy is given by the so-called game version of the Pontryagin principle, see for instance [5, Chap. 2]. In our setting, it says that any equilibrim must satisfy the following forward-backward system:

$$
\begin{aligned}
& d X_{t}^{i}=-Y_{t}^{i} d t+d W_{t}^{i}, \\
& d Y_{t}^{i}=-\left(X_{t}^{i}-\int_{\mathbb{R}} f(x) d \bar{\mu}_{t}^{N, i}(x)\right) d t+\sum_{j=1}^{N} Z_{t}^{i, j} d W_{t}^{j},
\end{aligned}
$$

for $t \in[0, T]$ and $i=1, \cdots, N$, with the terminal boundary condition

$$
Y_{T}^{i}=X_{T}^{i}-\int_{\mathbb{R}} g(x) d \bar{\mu}_{T}^{N, i}(x), \quad i=1, \cdots, N .
$$

As already explained, the solutions of the backward equations are required to be adapted and the martingale integrands to be square-integrable. By using the same kind of change of variable as in Section 2, we can "remove" the linear term in $x$ in the backward component; thanks to [9], we can deduce that the above system is uniquely solvable when $f$ and $g$ are bounded and Lipschitz continuous.

Following the strategy introduced in [3], we let

$$
\bar{Y}_{t}^{i}=\mathcal{U}\left(t, X_{t}^{i}, \bar{\mu}_{t}^{N}\right), \quad \bar{Z}_{t}^{i}=\partial_{x} \mathcal{U}\left(t, X_{t}^{i}, \bar{\mu}_{t}^{N}\right)=1, \quad t \in[0, T]
$$

for $i \in\{1, \cdots, N\}$, where $\mathcal{U}$ is given by (7) and with

$$
\bar{\mu}_{t}^{N}=\frac{1}{N} \sum_{j=1}^{N} \delta_{X_{t}^{j}}, \quad t \in[0, T] .
$$

Pay attention to make a distinction between $\bar{\mu}_{t}^{N}$, which is the empirical distribution of the whole system, and $\bar{\mu}_{t}^{N, i}$, which is the empirical distribution of the players connected to $i$.

The main strategy for addressing the convergence of the solution to (9) (as $N$ tends to $\infty$ ) is to consider the distance between $\left(\bar{Y}_{t}^{i}\right)_{0 \leqslant t \leqslant T}$ and $\left(Y_{t}^{i}\right)_{0 \leqslant t \leqslant T}$, which is the purpose of the following claim. 
Proposition 6. There exists a constant $C$, independent of $N$ and of the realization of the Erdös-Renyi graph, such that

$$
\begin{aligned}
& \sup _{0 \leqslant t \leqslant T}\left(\frac{1}{N} \sum_{i=1}^{N} \mathbb{E}\left[\left|\bar{Y}_{t}^{i}-Y_{t}^{i}\right|^{2}\right]\right) \\
& \leqslant \frac{C}{N^{2}}+C \frac{1}{N} \sum_{i=1}^{N} \mathbb{E}\left[\left(\frac{1}{N_{i}} \mathbf{1}_{N_{i} \geqslant 1} \sum_{j=1}^{N}\left(\varepsilon^{i, j} g\left(X_{T}^{j}\right)\right)-\frac{1}{N} \sum_{j=1}^{N} g\left(X_{T}^{j}\right)\right)^{2}\right] \\
& \quad+C \int_{0}^{T} \frac{1}{N} \sum_{i=1}^{N} \mathbb{E}\left[\left(\frac{1}{N_{i}} \mathbf{1}_{N_{i} \geqslant 1} \sum_{j=1}^{N}\left(\varepsilon^{i, j} f\left(X_{t}^{j}\right)\right)-\frac{1}{N} \sum_{j=1}^{N} f\left(X_{t}^{j}\right)\right)^{2}\right] \tilde{A} \succeq d t .
\end{aligned}
$$

As made clear below, the proof is based on the standard Itô formula, applied to the function:

$$
\begin{aligned}
& u^{i}:[0, T] \times \mathbb{R}^{d} \ni(t, \boldsymbol{x})=\left(t, x^{1}, \cdots, x^{N}\right) \mapsto \mathcal{U}\left(t, x^{i}, \bar{\mu}_{(\boldsymbol{x})}^{N}\right), \\
& \text { with } \quad \bar{\mu}_{(\boldsymbol{x})}^{N}=\frac{1}{N} \sum_{j=1}^{N} \delta_{x^{j}} .
\end{aligned}
$$

Here again, pay attention to distinguish $\bar{\mu}_{(\boldsymbol{x})}^{N}$ from $\bar{\mu}_{t}^{N}$. The first one is indexed by a vector $\boldsymbol{x} \in \mathbb{R}^{N}$, whilst the second one is indexed by a time instant $t \in[0, T]$.

In order to proceed, we must explain how to compute the (Euclidean) derivatives of $u^{i}$ in terms of the Wasserstein derivative of $\mathcal{U}$. The following identities are taken from [3,8] or [5].

$$
\begin{aligned}
\partial_{x^{i}} u^{i}\left(t, x^{1}, \cdots, x^{N}\right)= & \partial_{x} \mathcal{U}\left(t, x^{i}, \bar{\mu}_{(\boldsymbol{x})}^{N}\right)+\frac{1}{N} \partial_{\mu} \mathcal{U}\left(t, x^{i}, \bar{\mu}_{(\boldsymbol{x})}^{N}\right)\left(x^{i}\right) \\
\partial_{x^{j}} u^{i}\left(t, x^{1}, \cdots, x^{N}\right)= & \frac{1}{N} \partial_{\mu} \mathcal{U}\left(t, x^{i}, \bar{\mu}_{(\boldsymbol{x})}^{N}\right)\left(x^{j}\right), \quad j \neq i \\
\partial_{x^{i}}^{2} u^{i}\left(t, x^{1}, \cdots, x^{N}\right)= & \partial_{x}^{2} \mathcal{U}\left(t, x^{i}, \bar{\mu}_{(\boldsymbol{x})}^{N}\right)+\frac{1}{N} \partial_{v} \partial_{\mu} \mathcal{U}\left(t, x^{i}, \bar{\mu}_{(\boldsymbol{x})}^{N}\right)\left(x^{i}\right) \\
& +\frac{1}{N^{2}} \partial_{\mu}^{2} \mathcal{U}\left(t, x^{i}, \bar{\mu}_{(\boldsymbol{x})}^{N}\right)\left(x^{i}, x^{i}\right) \\
= & \frac{1}{N} \partial_{v} \partial_{\mu} \mathcal{U}\left(t, x^{i}, \bar{\mu}_{(\boldsymbol{x})}^{N}\right)\left(x^{i}\right)+\frac{1}{N^{2}} \partial_{\mu}^{2} \mathcal{U}\left(t, x^{i}, \bar{\mu}_{(\boldsymbol{x})}^{N}\right)\left(x^{i}, x^{i}\right),
\end{aligned}
$$

and

$$
\begin{aligned}
\partial_{x^{j}}^{2} u^{i}\left(t, x^{1}, \cdots, x^{N}\right)= & \frac{1}{N} \partial_{v} \partial_{\mu} \mathcal{U}\left(t, x^{i}, \bar{\mu}_{(\boldsymbol{x})}^{N}\right)\left(x^{j}\right) \\
& +\frac{1}{N^{2}} \partial_{\mu}^{2} \mathcal{U}\left(t, x^{i}, \bar{\mu}_{(\boldsymbol{x})}^{N}\right)\left(x^{j}, x^{j}\right), \quad j \neq i,
\end{aligned}
$$

where, to compute the second-order derivatives, we used the fact that, in our case, $\partial_{x} \mathcal{U}(t, x, \mu)$ is constant (equal to 1 ) and $\partial_{\mu} \mathcal{U}(t, x, \mu)(v)$ is independent of $x$. 
Proof. By applying Itô's formula and by implementing the PDE satisfied by $\mathcal{U}$ at point $\left(t, x^{i}, \bar{\mu}_{(\boldsymbol{x})}^{N}\right)$, we get

$$
\begin{aligned}
d \bar{Y}_{t}^{i}= & \partial_{x} \mathcal{U}\left(t, X_{t}^{i}, \bar{\mu}_{t}^{N}\right)\left(\mathcal{U}\left(t, X_{t}^{i}, \bar{\mu}_{t}^{N}\right)-Y_{t}^{i}\right) d t \\
& +\frac{1}{N} \sum_{j=1}^{N} \partial_{\mu} \mathcal{U}\left(t, X_{t}^{i}, \bar{\mu}_{t}^{N}\right)\left(X_{t}^{j}\right)\left(\mathcal{U}\left(t, X_{t}^{j}, \bar{\mu}_{t}^{N}\right)-Y_{t}^{j}\right) d t \\
& +\frac{1}{2 N^{2}} \sum_{j=1}^{N} \partial_{\mu}^{2} \mathcal{U}\left(t, X_{t}^{i}, \bar{\mu}_{t}^{N}\right)\left(X_{t}^{j}, X_{t}^{j}\right) d t \\
& -\left(X_{t}^{i}-\int_{\mathbb{R}} f(x) d \bar{\mu}_{t}^{N}(x)\right) d t \\
& +\bar{Z}_{t}^{i} d W_{t}^{i}+\frac{1}{N} \sum_{j=1}^{N} \partial_{\mu} \mathcal{U}\left(t, X_{t}^{i}, \bar{\mu}_{t}^{N}\right)\left(X_{t}^{j}\right) d W_{t}^{j} .
\end{aligned}
$$

Inserting the backward equation satisfied by $\left(Y_{t}^{i}\right)_{0 \leqslant t \leqslant T}$ and recalling that $\left(\bar{Y}_{t}^{i}=\mathcal{U}\left(t, X_{t}^{i}, \bar{\mu}_{t}^{N}\right)\right)_{0 \leqslant t \leqslant T}$, we obtain

$$
\begin{aligned}
d\left(\bar{Y}_{t}^{i}-Y_{t}^{i}\right)= & \partial_{x} \mathcal{U}\left(t, X_{t}^{i}, \bar{\mu}_{t}^{N}\right)\left(\bar{Y}_{t}^{i}-Y_{t}^{i}\right) d t \\
& +\frac{1}{N} \sum_{j=1}^{N} \partial_{\mu} \mathcal{U}\left(t, X_{t}^{i}, \bar{\mu}_{t}^{N}\right)\left(X_{t}^{j}\right)\left(\bar{Y}_{t}^{j}-Y_{t}^{j}\right) d t \\
& +\frac{1}{2 N^{2}} \sum_{j=1}^{N} \partial_{\mu}^{2} \mathcal{U}\left(t, X_{t}^{i}, \bar{\mu}_{t}^{N}\right)\left(X_{t}^{j}, X_{t}^{j}\right) d t \\
& -\left(\frac{1}{N_{i}} \sum_{j=1}^{N} \varepsilon^{i, j} f\left(X_{t}^{j}\right)-\frac{1}{N} \sum_{j=1}^{N} f\left(X_{t}^{j}\right)\right) d t \\
& +\sum_{j=1}^{N}\left(\bar{Z}_{t}^{i} \mathbf{1}_{i=j}+\frac{1}{N} \partial_{\mu} \mathcal{U}\left(t, X_{t}^{i}, \bar{\mu}_{t}^{N}\right)\left(X_{t}^{j}\right)-Z_{t}^{i, j}\right) d W_{t}^{j}
\end{aligned}
$$

Taking the square and then the expectation, we deduce that there exists a constant $C$, independent of $N$ and $i$, such that, for all $t \in[0, T]$,

$$
\begin{aligned}
d \mathbb{E}\left[\left|\bar{Y}_{t}^{i}-Y_{t}^{i}\right|^{2}\right] \leqslant & C \mathbb{E}\left[\left|\bar{Y}_{t}^{i}-Y_{t}^{i}\right|^{2}\right] d t \\
& +C \frac{1}{N} \sum_{j=1}^{N} \mathbb{E}\left[\left|\bar{Y}_{t}^{j}-Y_{t}^{j}\right|^{2}\right] d t \\
& +C\left\{\frac{1}{N^{2}}+\mathbb{E}\left[\left(\frac{1}{N_{i}} \sum_{j=1}^{N} \varepsilon^{i, j} f\left(X_{t}^{j}\right)-\frac{1}{N} \sum_{j=1}^{N} f\left(X_{t}^{j}\right)\right)^{2}\right]\right\} \tilde{\mathrm{A}} \succeq d t .
\end{aligned}
$$

where we used the fact that the derivatives of $\mathcal{U}$ are bounded. 
Recalling that $\bar{Y}_{T}^{i}=\mathcal{U}\left(T, X_{T}^{i}, \bar{\mu}_{T}^{N}\right)=X_{T}^{i}-\int_{\mathbb{R}} g(x) d \bar{\mu}_{T}^{N}(x)$, taking the mean over $i \in\{1, \ldots, N\}$ and appealing to Gronwall's lemma, we obtain

$$
\begin{aligned}
& \sup _{0 \leqslant t \leqslant T}\left(\frac{1}{N} \sum_{i=1}^{N} \mathbb{E}\left[\left|\bar{Y}_{t}^{i}-Y_{t}^{i}\right|^{2}\right]\right) \\
& \leqslant \frac{C}{N^{2}}+C \frac{1}{N} \sum_{i=1}^{N} \mathbb{E}\left[\left(\frac{1}{N_{i}} \sum_{j=1}^{N}\left(\varepsilon^{i, j} g\left(X_{T}^{j}\right)\right)-\frac{1}{N} \sum_{j=1}^{N} g\left(X_{T}^{j}\right)\right)^{2}\right] \\
& \quad+C \int_{0}^{T} \frac{1}{N} \sum_{i=1}^{N} \mathbb{E}\left[\left(\frac{1}{N_{i}} \sum_{j=1}^{N}\left(\varepsilon^{i, j} f\left(X_{t}^{j}\right)\right)-\frac{1}{N} \sum_{j=1}^{N} f\left(X_{t}^{j}\right)\right)^{2}\right] \tilde{\mathrm{A}} \succeq d t
\end{aligned}
$$

which completes the proof.

\subsection{Almost sure analysis on the graph}

We now compute (with a similar computation for the term driven by $f$ ):

$$
\begin{aligned}
& \frac{1}{N} \sum_{i=1}^{N} \mathbb{E}\left[\left(\frac{1}{N_{i}} \sum_{j=1}^{N}\left(\varepsilon^{i, j} g\left(X_{T}^{j}\right)\right)-\frac{1}{N} \sum_{j=1}^{N} g\left(X_{T}^{j}\right)\right)^{2}\right] \\
& =\mathbb{E}\left[\frac{1}{N} \sum_{i=1}^{N}\left(\frac{\mathbf{1}_{N_{i} \geqslant 1}}{N_{i}^{2}} \sum_{j, \ell=1}^{N}\left(\varepsilon^{i, j} \varepsilon^{i, \ell} g\left(X_{T}^{j}\right) g\left(X_{T}^{\ell}\right)\right)+\frac{1}{N^{2}} \sum_{j, \ell=1}^{N} g\left(X_{T}^{j}\right) g\left(X_{T}^{\ell}\right)\right)\right] \\
& \quad-\frac{2}{N^{2}} \mathbb{E}\left[\sum_{i=1}^{N} \frac{\mathbf{1}_{N_{i} \geqslant 1}}{N_{i}} \sum_{j, \ell=1}^{N}\left(\varepsilon^{i, j} g\left(X_{T}^{j}\right) g\left(X_{T}^{\ell}\right)\right)\right]
\end{aligned}
$$

Observe that

$$
\begin{aligned}
& \frac{1}{N} \sum_{i=1}^{N} \frac{\mathbf{1}_{N_{i} \geqslant 1}}{N_{i}^{2}} \sum_{j, \ell=1}^{N}\left(\varepsilon^{i, j} \varepsilon^{i, \ell} g\left(X_{T}^{j}\right) g\left(X_{T}^{\ell}\right)\right) \\
& =\frac{1}{N} \sum_{j, \ell=1}^{N}\left[\left(\sum_{i=1}^{N} \frac{\mathbf{1}_{N_{i} \geqslant 1}}{N_{i}^{2}} \varepsilon^{i, j} \varepsilon^{i, \ell}\right) g\left(X_{T}^{j}\right) g\left(X_{T}^{\ell}\right)\right] \\
& =\frac{1}{N^{2}} \sum_{j, \ell=1}^{N}\left[\left(\frac{1}{N} \sum_{i=1}^{N} \frac{N^{2}}{N_{i}^{2}} \mathbf{1}_{N_{i} \geqslant 1} \varepsilon^{i, j} \varepsilon^{i, \ell}\right) g\left(X_{T}^{j}\right) g\left(X_{T}^{\ell}\right)\right]
\end{aligned}
$$


and,

$$
\begin{aligned}
& \frac{2}{N^{2}} \sum_{i=1}^{N} \frac{\mathbf{1}_{N_{i} \geqslant 1}}{N_{i}} \sum_{j, \ell=1}^{N}\left(\varepsilon^{i, j} g\left(X_{T}^{j}\right) g\left(X_{T}^{\ell}\right)\right) \\
& =\frac{2}{N^{2}} \sum_{j, \ell=1}^{N}\left[\left(\sum_{i=1}^{N} \frac{\mathbf{1}_{N_{i} \geqslant 1}}{N_{i}} \varepsilon^{i, j}\right) g\left(X_{T}^{j}\right) g\left(X_{T}^{\ell}\right)\right] \\
& =\frac{2}{N^{2}} \sum_{j, \ell=1}^{N}\left[\left(\frac{1}{N} \sum_{i=1}^{N} \frac{N}{N_{i}} \mathbf{1}_{N_{i} \geqslant 1} \varepsilon^{i, j}\right) g\left(X_{T}^{j}\right) g\left(X_{T}^{\ell}\right)\right] .
\end{aligned}
$$

We claim:

Proposition 7. On $(\Xi, \mathcal{G}, \mathbf{P})$, consider a sequence $\left(\varepsilon^{i, j}\right)_{j>i \geqslant 1}$ of independent and identically distributed Bernoulli random variables of parameter $p \in(0,1)$. Let $\varepsilon^{i, i}=0$ and $\varepsilon^{i, j}=\varepsilon^{j, i}$ if $i>j$.

Then, $\mathbf{P}$ almost surely,

$$
\begin{aligned}
& \lim _{N \rightarrow \infty} \frac{1}{N^{2}} \sum_{j, \ell=1}^{N}\left|1-\frac{1}{N} \sum_{i=1}^{N} \frac{N^{2}}{N_{i}^{2}} \mathbf{1}_{N_{i} \geqslant 1} \varepsilon^{i, j} \varepsilon^{i, \ell}\right|=0, \\
& \lim _{N \rightarrow \infty} \frac{1}{N} \sum_{j=1}^{N}\left|1-\frac{1}{N} \sum_{i=1}^{N} \frac{N}{N_{i}} \mathbf{1}_{N_{i} \geqslant 1} \varepsilon^{i, j}\right|=0
\end{aligned}
$$

where

$$
N_{i}=\frac{1}{N} \sum_{j=1}^{N} \varepsilon^{i, j}
$$

As the proof of Proposition 7 could distract the reader from the main line of the text, it is postponed to the appendix below.

\subsection{Final statement}

From now on, we assume that $(\Omega, \mathcal{F}, \mathbb{P})$ is equipped with an infinite sequence of independent Brownian motions $\left(\left(W_{t}^{i}\right)_{0 \leqslant t \leqslant T}\right)_{i \geqslant 1}$ and with an infinite sequence of identically distributed and independent initial conditions $\left(X_{0}^{i}\right)_{i \geqslant 1}$, both sequences being required to be independent.

Here is then the final statement.

Theorem 1. Assume that $f$ and $g$ are bounded and Lipschitz continuous and that the conclusion of Proposition 4 holds true.

On $(\Xi, \mathcal{G}, \mathbf{P})$, consider a sequence $\left(\varepsilon^{i, j}\right)_{j>i \geqslant 1}$ of independent and identically distributed Bernoulli random variables of parameter $p \in(0,1)$. Let $\varepsilon^{i, i}=0$ and $\varepsilon^{i, j}=\varepsilon^{j, i}$ if $i>j$.

Then, $\mathbf{P}$ almost surely,

$$
\lim _{N \rightarrow \infty} \sup _{0 \leqslant t \leqslant T} \mathbb{E}\left[\mathcal{W}_{2}\left(\bar{\mu}_{t}^{N}, \mu_{t}\right)^{2}\right]=0,
$$

where $\mathcal{W}_{2}$ denotes the 2 -Wasserstein distance on $\mathcal{P}_{2}(\mathbb{R}),\left(\bar{\mu}_{t}^{N}\right)_{0 \leqslant t \leqslant T}$ is as in $(10)$ and $\left(\mu_{t}\right)_{0 \leqslant t \leqslant T}$ is equal to $\left(\mathcal{L}\left(X_{t}\right)\right)_{0 \leqslant t \leqslant T},\left(X_{t}\right)_{0 \leqslant t \leqslant T}$ denoting the forward component of the solution to (1). 
Proof. By (12), (13) and (14), and by Proposition 7, we deduce that there exists a sequence of random variables $\left(\delta_{N}\right)_{N \geqslant 1}$ constructed on $(\Xi, \mathcal{G}, \mathbf{P})$ such that

$$
\mathbf{P}\left[\lim _{N \rightarrow \infty} \delta_{N}=0\right]=1,
$$

and

$$
\frac{1}{N} \sum_{i=1}^{N} \mathbb{E}\left[\left(\frac{1}{N_{i}} \sum_{j=1}^{N}\left(\varepsilon^{i, j} g\left(X_{T}^{j}\right)\right)-\frac{1}{N} \sum_{j=1}^{N} g\left(X_{T}^{j}\right)\right)^{2}\right] \leqslant \delta_{N} .
$$

Proceeding similarly with the terms driven by $f$ in (11), we deduce that

$$
\sup _{0 \leqslant t \leqslant T}\left(\frac{1}{N} \sum_{i=1}^{N} \mathbb{E}\left[\left|\bar{Y}_{t}^{i}-Y_{t}^{i}\right|^{2}\right]\right) \leqslant \delta_{N}
$$

the details of $\left(\delta_{N}\right)_{N \geqslant 1}$ being allowed to change from line to line.

For any $i \geqslant 1$, call $\left(\left(\hat{X}_{t}^{i}\right)_{0 \leqslant t \leqslant T}\right)_{i \geqslant 1}$ the copy of $\left(X_{t}\right)_{0 \leqslant t \leqslant T}$ when driven by $\left(X_{0}^{i},\left(W_{t}^{i}\right)_{0 \leqslant t \leqslant T}\right)$ instead of $\left(X_{0},\left(W_{t}\right)_{0 \leqslant t \leqslant T}\right)$, namely

$$
d \hat{X}_{t}^{i}=-\mathcal{U}\left(t, \hat{X}_{t}^{i}, \mathcal{L}\left(\hat{X}_{t}^{i}\right)\right) d t+d W_{t}^{i}, \quad t \in[0, T] ; \quad \hat{X}_{0}^{i}=X_{0}^{i} .
$$

Then, recalling that $\left(\bar{Y}_{t}^{i}=\mathcal{U}\left(t, X_{t}^{i}, \bar{\mu}_{t}^{N}\right)\right)_{0 \leqslant t \leqslant T}$ and using the Lipschitz property of $\mathcal{U}$ in $x$ and $\mu$, we have

$$
\mathbb{E}\left[\left|\hat{X}_{t}^{i}-X_{t}^{i}\right|^{2}\right] \leqslant C \int_{0}^{t} \mathbb{E}\left[\left|\bar{Y}_{s}^{i}-Y_{s}^{i}\right|^{2}\right] d s+C \int_{0}^{t} \mathbb{E}\left[\mathcal{W}_{2}\left(\bar{\mu}_{s}^{N}, \mu_{s}\right)^{2}\right] d s, \quad t \in[0, T],
$$

for a constant $C$ independent of $N$ and $t$. Above, we used the identity $\left(\mu_{t}=\mathcal{L}\left(\hat{X}_{t}^{i}\right)\right)_{0 \leqslant t \leqslant T}$. Taking the mean over $i$ and using (15), we obtain

$$
\mathbb{E}\left[\mathcal{W}_{2}\left(\bar{\mu}_{t}^{N}, \hat{\mu}_{t}^{N}\right)^{2}\right] \leqslant \delta_{N}+C \int_{0}^{t} \mathbb{E}\left[\mathcal{W}_{2}\left(\bar{\mu}_{s}^{N}, \mu_{s}\right)^{2}\right] d s, \quad t \in[0, T]
$$

where we let

$$
\hat{\mu}_{t}^{N}=\frac{1}{N} \sum_{i=1}^{N} \delta_{\hat{X}_{t}^{i}}, \quad t \in[0, T]
$$

This yields

$$
\sup _{0 \leqslant t \leqslant T} \mathbb{E}\left[\mathcal{W}_{2}\left(\bar{\mu}_{t}^{N}, \hat{\mu}_{t}^{N}\right)^{2}\right] \leqslant \delta_{N}+\int_{0}^{T} \mathbb{E}\left[\mathcal{W}_{2}\left(\hat{\mu}_{t}^{N}, \mu_{t}\right)^{2}\right] d t .
$$

As $\hat{\mu}_{t}^{N}$ in the right-hand side is the empirical distribution of an independent and identically distributed sample of law $\mu_{t}$, for each $t \in[0, T]$, the term $\mathbb{E}\left[\mathcal{W}_{2}\left(\hat{\mu}_{t}^{N}, \mu_{t}\right)^{2}\right]$ tends to 0 as $N$ tends to $\infty$. As $\sup _{0 \leqslant t \leqslant T} \mathbb{E}\left[\mathcal{W}_{2}\left(\hat{\mu}_{t}^{N}, \mu_{t}\right)^{2}\right] \leqslant 2 \sup _{0 \leqslant t \leqslant T} \mathbb{E}\left[\left|X_{t}\right|^{2}\right]<\infty$, Lebesgue's dominated convergence theorem shows that the integral tends to 0 as well. Hence,

$$
\lim _{N \rightarrow \infty} \sup _{0 \leqslant t \leqslant T} \mathbb{E}\left[\mathcal{W}_{2}\left(\bar{\mu}_{t}^{N}, \hat{\mu}_{t}^{N}\right)^{2}\right]=0
$$


It remains to observe that, in fact,

$$
\lim _{N \rightarrow \infty} \sup _{0 \leqslant t \leqslant T} \mathbb{E}\left[\mathcal{W}_{2}\left(\hat{\mu}_{t}^{N}, \mu_{t}\right)^{2}\right]=0
$$

which follows from a uniform continuity argument. Indeed, for any $0 \leqslant s<t \leqslant T$,

$$
\begin{aligned}
\mathbb{E}\left[\left|\mathcal{W}_{2}\left(\hat{\mu}_{t}^{N}, \mu_{t}\right)^{2}-\mathcal{W}_{2}\left(\hat{\mu}_{s}^{N}, \mu_{s}\right)^{2}\right|\right] & \leqslant C \mathbb{E}\left[\left|\mathcal{W}_{2}\left(\hat{\mu}_{t}^{N}, \mu_{t}\right)-\mathcal{W}_{2}\left(\hat{\mu}_{s}^{N}, \mu_{s}\right)\right|^{2}\right]^{1 / 2} \\
& \leqslant C \mathbb{E}\left[\mathcal{W}_{2}\left(\hat{\mu}_{t}^{N}, \hat{\mu}_{s}^{N}\right)^{2}+\mathcal{W}_{2}\left(\mu_{t}, \mu_{s}\right)^{2}\right]^{1 / 2} \\
& \leqslant C \mathbb{E}\left[\left|X_{t}-X_{s}\right|^{2}\right] \\
& \leqslant C(t-s)
\end{aligned}
$$

the constant $C$ being allowed to vary from line to line.

\subsection{Appendix}

We now prove the statement of Proposition 7.

Proof. Fix $N \geqslant 2$ and observe, for a given $i \in\{1, \cdots, N\}$, that the random variables $\left(\varepsilon^{i, j}\right)_{j \neq i}$ are independent and identically distributed. By Hoeffding's inequality,

$$
\forall \varepsilon>0, \quad \mathbf{P}\left[\left|\frac{N_{i}}{N-1}-p\right| \geqslant \varepsilon\right] \leqslant 2 \exp \left(-2(N-1) \varepsilon^{2}\right)
$$

First Step. By (16), we have

$$
\forall t>0, \quad \mathbf{P}\left[\sqrt{N-1}\left|\frac{N_{i}}{N-1}-p\right| \geqslant t\right] \leqslant 2 \exp \left(-2 t^{2}\right)
$$

from which we deduce that, for any integer $q \geqslant 1$,

$$
\mathbf{E}\left[\left|\frac{N_{i}}{N-1}-p\right|^{q}\right] \leqslant \frac{C_{q}}{N^{q / 2}},
$$

for a constant $C_{q}$ only depending on $q$. Hence,

$$
\begin{aligned}
\mathbf{E}\left[\left|\frac{N_{i}}{N}-p\right|^{q}\right] & \leqslant 2^{q-1} \mathbf{E}\left[\left|\frac{N_{i}}{N-1}-p\right|^{q}\right]+2^{q-1} \mathbf{E}\left[\left|\frac{N_{i}}{N}-\frac{N_{i}}{N-1}\right|^{q}\right] \\
& \leqslant \frac{C_{q}}{N^{q / 2}},
\end{aligned}
$$

where we allowed the constant $C_{q}$ to vary from line to line. 
Second Step. By (16), we also have, for any large enough real $k \geqslant 1$, precisely for $1 / k \leqslant p / 2$,

$$
\begin{aligned}
\mathbf{P}\left[\frac{N-1}{N_{i}} \mathbf{1}_{N_{i} \geqslant 1} \geqslant k\right] & \leqslant \mathbf{P}\left[\frac{N_{i}}{N-1} \leqslant \frac{1}{k}\right] \\
& \leqslant \mathbf{P}\left[\left|\frac{N_{i}}{N-1}-p\right| \geqslant \frac{p}{2}\right] \\
& \leqslant 2 \exp \left(-\frac{(N-1) p^{2}}{2}\right) .
\end{aligned}
$$

In particular, for any integer $q \geqslant 1$,

$$
\begin{aligned}
\mathbf{E}\left[\left(\frac{N-1}{N_{i}}\right)^{q} \mathbf{1}_{N_{i} \geqslant 1}\right] & =q \int_{0}^{N} k^{q-1} \mathbf{P}\left[\frac{N-1}{N_{i}} \mathbf{1}_{N_{i} \geqslant 1} \geqslant k\right] d k \\
& \leqslant q \int_{0}^{2 / p} k^{q-1} d k+2 q \int_{0}^{N} k^{q-1} \exp \left(-\frac{(N-1) p^{2}}{2}\right) d k \\
& \leqslant \frac{2^{q}}{p^{q}}+2 N^{q} \exp \left(-\frac{(N-1) p^{2}}{2}\right) .
\end{aligned}
$$

Allowing the constant $C_{q}$ to depend on $p$, we obtain

$$
\mathbf{E}\left[\left(\frac{N}{N_{i}}\right)^{q} \mathbf{1}_{N_{i} \geqslant 1}\right] \leqslant C_{q}
$$

By (17) and (18) and by Cauchy-Schwarz inequality,

$$
\begin{aligned}
\mathbf{E}\left[\left|\frac{N}{N_{i}} \mathbf{1}_{N_{i} \geqslant 1}-\frac{1}{p}\right|^{q}\right] & =\mathbf{E}\left[\left(\frac{N \mathbf{1}_{N_{i}} \geqslant 1}{p N_{i}}\right)^{q}\left|p-\frac{N_{i}}{N}\right|^{q}\right]+\frac{1}{p^{q}} \mathbf{P}\left[N_{i}=0\right] \\
& \leqslant \mathbf{E}\left[\left(\frac{N \mathbf{1}_{N_{i} \geqslant 1}}{p N_{i}}\right)^{q}\left|p-\frac{N_{i}}{N}\right|^{q}\right]+\frac{1}{p^{q}} \mathbf{P}\left[\left|\frac{N_{i}}{N-1}-p\right| \geqslant p\right] \\
& \leqslant \frac{C_{q}}{N^{q / 2}}
\end{aligned}
$$

where we made use of (16) again to derive the last term in the second line.

Third Step. We now prove the second claim in the statement of Proposition 7.

$$
\begin{aligned}
& \frac{1}{N} \sum_{j=1}^{N}\left|1-\frac{1}{N} \sum_{i=1}^{N} \frac{N}{N_{i}} \mathbf{1}_{N_{i} \geqslant 1} \varepsilon^{i, j}\right| \\
& \leqslant \frac{1}{N} \sum_{j=1}^{N}\left|\frac{1}{N} \sum_{i=1}^{N}\left(\frac{N}{N_{i}} \mathbf{1}_{N_{i} \geqslant 1}-\frac{1}{p}\right) \varepsilon^{i, j}\right|+\frac{1}{p N} \sum_{j=1}^{N}\left|\frac{N_{j}}{N}-p\right| \\
& \leqslant \frac{1}{N} \sum_{i=1}^{N}\left|\frac{N}{N_{i}} \mathbf{1}_{N_{i} \geqslant 1}-\frac{1}{p}\right|+\frac{1}{p N} \sum_{j=1}^{N}\left|\frac{N_{j}}{N}-p\right| .
\end{aligned}
$$


Taking the power $q$ and then the expectation under $\mathbf{P}$ and appealing to the conclusions of the two first steps, we get

$$
\mathbf{E}\left[\left(\frac{1}{N} \sum_{j=1}^{N}\left|1-\frac{1}{N} \sum_{i=1}^{N} \frac{N}{N_{i}} \mathbf{1}_{N_{i} \geqslant 1} \varepsilon^{i, j}\right|\right)^{q}\right] \leqslant \frac{C_{q}}{N^{q / 2}}
$$

which suffices to conclude.

Fourth Step. In order to tackle the first claim, we first observe that

$$
\mathbf{E}\left[\left|\frac{N^{2}}{N_{i}^{2}} \mathbf{1}_{N_{i} \geqslant 1}-\frac{1}{p^{2}}\right|^{q}\right] \leqslant \mathbf{E}\left[\left|\frac{N}{N_{i}} \mathbf{1}_{N_{i} \geqslant 1}+\frac{1}{p}\right|^{2 q}\right]^{1 / 2} \mathbf{E}\left[\left|\frac{N}{N_{i}} \mathbf{1}_{N_{i} \geqslant 1}-\frac{1}{p}\right|^{2 q}\right]^{1 / 2} .
$$

By (18) and the conclusion of the second step, we deduce that

$$
\mathbf{E}\left[\left|\frac{N^{2}}{N_{i}^{2}} \mathbf{1}_{N_{i} \geqslant 1}-\frac{1}{p^{2}}\right|^{q}\right] \leqslant \frac{C_{q}}{N^{q / 2}}
$$

We also note that, for any given $j \neq \ell$, the random variables $\left(\varepsilon^{i, \ell} \varepsilon^{i, j}\right)_{i \neq j, i \neq \ell}$ are independent and follow the same Bernoulli distribution of parameter $p^{2}$. Therefore, proceeding as in the first step, we have

$$
\mathbf{E}\left[\left|\frac{1}{N} \sum_{i=1}^{N} \varepsilon^{i, \ell} \varepsilon^{i, j}-p^{2}\right|^{q}\right] \leqslant \frac{C_{q}}{N^{q / 2}}
$$

In order to complete the proof, it remains to see, in analogy with the third step, that

$$
\begin{aligned}
& \frac{1}{N^{2}} \sum_{j, \ell=1}^{N}\left|1-\frac{1}{N} \sum_{i=1}^{N} \frac{N^{2}}{N_{i}^{2}} \mathbf{1}_{N_{i} \geqslant 1} \varepsilon^{i, j} \varepsilon^{i, \ell}\right| \\
& \leqslant \frac{1}{N} \sum_{i=1}^{N}\left|\frac{N^{2}}{N_{i}^{2}} \mathbf{1}_{N_{i} \geqslant 1}-\frac{1}{p^{2}}\right|+\frac{1}{N^{2} p^{2}} \sum_{j, \ell=1}^{N}\left|p^{2}-\frac{1}{N} \sum_{i=1}^{N} \varepsilon^{i, j} \varepsilon^{i, \ell}\right| .
\end{aligned}
$$

Taking the power $q$ and then the expectation under $\mathbf{P}$,

$$
\mathbf{E}\left[\left(\frac{1}{N^{2}} \sum_{j, \ell=1}^{N}\left|1-\frac{1}{N} \sum_{i=1}^{N} \frac{N^{2}}{N_{i}^{2}} \mathbf{1}_{N_{i} \geqslant 1} \varepsilon^{i, j} \varepsilon^{i, \ell}\right|\right)^{q}\right] \leqslant \frac{C_{q}}{N^{q / 2}}
$$

which yields the result.

\section{Acknowledgement}

The author is especially grateful to the organizers of the "Journées MAS 2016" which were held in Grenoble, and in particular to the local organizers Jean-François Coeurjolly and Adeline Samson and to the president of the scientific committee, Francis Comets.

The author also thanks to the two anonymous referees for their valuable comments on the paper. 


\section{REFERENCES}

[1] A. Bensoussan, J. Frehse, and P. Yam. Mean Field Games and Mean Field Type Control Theory. Springer Briefs in Mathematics. Springer Verlag, 2013.

[2] P. Cardaliaguet. Notes from P.L. Lions' lectures at the Collège de France. Technical report, https://www.ceremade.dauphine.fr/ cardalia/MFG100629.pdf, 2012.

[3] P. Cardaliaguet, F. Delarue, J.-M. Lasry, and P.-L. Lions. The master equation and the convergence problem in mean field games. Technical report, http://arxiv.org/abs/1509.02505, 2015.

[4] R. Carmona and F. Delarue. Probabilistic analysis of mean field games. SIAM Journal on Control and Optimization, 51:2705-2734, 2013.

[5] R. Carmona and F. Delarue. Probabilistic Theory of Mean Field Games: vol. I, Mean Field FBSDEs, Control, and Games. Stochastic Analysis and Applications. Springer Verlag, 2017.

[6] R. Carmona and F. Delarue. Probabilistic Theory of Mean Field Games: vol. II, Mean Field Games with Common Noise and Master Equations. Stochastic Analysis and Applications. Springer Verlag, 2017.

[7] R. Carmona, J.P. Fouque, and L.H. Sun. Mean field games and systemic risk: a toy model. Communications in Mathematical Sciences, 13:911-933, 2015.

[8] J.F. Chassagneux, D. Crisan, and F. Delarue. McKean-vlasov FBSDEs and related master equation. Technical report, http://arxiv.org/abs/1411.3009, 2015.

[9] F. Delarue. On the existence and uniqueness of solutions to FBSDEs in a non-degenerate case. Stochastic Processes and Applications, 99:209-286, 2002.

[10] M. Fischer. On the connection between symmetric $N$-player games and mean field games. Technical report, 2014.

[11] M. Huang, P.E. Caines, and R.P. Malhamé. Large population stochastic dynamic games: closed-loop McKean-Vlasov systems and the Nash certainty equivalence principle. Communications in Information and Systems, 6:221-252, 2006.

[12] M. Huang, P.E. Caines, and R.P. Malhamé. Large population cost coupled LQG problems with nonuniform agents: individual mass behavior and decentralized $\epsilon$-Nash equilibria. IEEE Transactions on Automatic Control, 52:1560-1571, 2007.

[13] D. Lacker. A general characterization of the mean field limit for stochastic differential games. Probability Theory and Related Fields, 165:581-648, 2016.

[14] J.M. Lasry and P.L. Lions. Jeux à champ moyen I. Le cas stationnaire. Comptes Rendus de l'Académie des Sciences de Paris, ser. A, 343(9), 2006.

[15] J.M. Lasry and P.L. Lions. Jeux à champ moyen II. Horizon fini et contrôle optimal. Comptes Rendus de l'Académie des Sciences de Paris, ser. A, 343(10), 2006.

[16] J.M. Lasry and P.L. Lions. Mean field games. Japanese Journal of Mathematics, 2(1):229-260, 2007.

[17] P.L. Lions. Théorie des jeux à champs moyen et applications. Lectures at the Collège de France. http://www.college-de-france.fr/default/EN/all/equ_der/cours_et_seminaires.htm, 2007-2008.

[18] J. Nash. Equilibrium points in n-person games. Proceedings National Academy of Sciences USA, 36:48-49, 1950.

[19] J. Nash. Non-cooperative games. Annals of Mathematics, 54:286-295, 1951.

[20] S. Peng and Z. Wu. Fully coupled forward-backward stochastic differential equations and applications to optimal control. SIAM Journal on Control and Optimization, 37:825-843, 1999.

[21] A.S. Sznitman. Topics in propagation of chaos. In D. L. Burkholder et al. , Ecole de Probabilités de Saint Flour, XIX-1989, volume 1464 of Lecture Notes in Mathematics, pages 165-251, 1989. 